\title{
Angular momentum dynamics and the intrinsic drift of monopolar vortices on a rotating sphere
}

\author{
Ramses van der Toorn ${ }^{1, a)}$ and Joseph T. F. Zimmerman ${ }^{2, b)}$ \\ ${ }^{1}$ Faculty Electrical Engineering, Mathematics and Computer Science, Delft University of \\ Technology, Mekelweg 4, 2628CD Delft, The Netherlands \\ ${ }^{2}$ Netherlands Institute for Sea Research, P.O. Box 59, Den Burg, 1790 AB Texel, The \\ Netherlands
}

(Received 3 July 2009; accepted 27 May 2010; published online 30 August 2010)

On the basis of the angular momentum equation for a fluid shell on a rotating planet, we analyze the intrinsic drift of a monopolar vortex in the shell. Central is the development of a general angular momentum equation for Eulerian fluid mechanics based on coordinate-free, general tensorial representations of the underlying fluid dynamics on the one hand, and an appropriate representation of the Lie algebra so(3) of rotations on the other hand. We show that angular momentum fluid dynamics concisely describes the motion of vortices along the sphere and explains why both geostrophic cyclones and anticyclones drift in retrograde direction (westward), why anticyclones do so faster than cyclones, and why this difference is enhanced by a cyclostrophic correction. Technically, the analysis is based on a tensorial representation of the integral angular momentum equation for the fluid shell as a whole, and, derived from this, a coordinate representation with respect to coordinates which may move with the vortex along the surface of the planet. Depicting the angular momentum balance of cyclones and anticyclones in terms of vector diagrams, we present an overview of the results achieved. () 2010 American Institute of Physics. [doi:10.1063/1.3455315]

\section{INTRODUCTION}

Large monopolar vortices (or "eddies") are ubiquitous phenomena in the fluid envelopes of rotating celestial bodies. This applies evidently to the Earth, where the most prominent examples are the mesoscale eddies or "rings" in the ocean ${ }^{1-3}$ and the tropical cyclones in the atmosphere, as it does to the visible outer layers of the giant planets, such as Jupiter's Great Red Spot and Neptune's Dark Spot. ${ }^{6,7}$ It may also apply to rapidly rotating stars, particularly neutron stars. ${ }^{8}$ All these vortices arise in one way or another from hydro- or thermodynamical instabilities. Once formed, the fate of a vortex is determined by its internal evolution and by its interaction with the ambient fluid, both of which affect its stability. If it is sufficiently stable, the timescale of its subsequent decay may be orders of magnitude larger than that of its formation or its rotation, ${ }^{9}$ so that they are "long lived" and "shape preserving." This applies, for instance, to mesoscale ocean eddies and Jupiter's Red Spot and its smaller (anti)cyclonic vortices. ${ }^{10,11}$ Tropical cyclones on the other hand have a relatively short formation time, a substantial internal development, and hardly a time interval over which they are in a stationary state.

Apart from its spinning motion a vortex as a whole may move relative to the solid body rotation of the planet or star. This movement may be passive if the vortex is carried along by the circulation of the ambient fluid. However, there is ample evidence that monopolar vortices in principle also always have an intrinsic retrograde drift, independently of the velocity of the

\footnotetext{
a)Electronic mail: ramses.vdToorn@inter.nl.net.

${ }^{b)}$ Electronic mail: zimmerman @introweb.nl. Also at Institute for Marine and Atmospheric Research, Utrecht University, The Netherlands.
} 
ambient fluid, although this drift may be masked by the drift due to the ambient fluid or by arresting of the vortex in the shear of the ambient fluid as seems to be the case for Jupiter's Red Spot. This intrinsic drift is the subject of this paper.

We present a theory of the intrinsic drift of monopolar vortices in a shallow fluid shell on a rotating sphere based on considerations of integral angular momentum. As the central areas of the mid latitude oceans on Earth are among the quietest regions in terms of external drift caused by the general circulation, it is not surprising that evidence of (westerly=retrograde) intrinsic drift comes primarily from these regions, where mesoscale eddies (or "rings") are shed to the quiet interior by strong boundary currents such as the Gulf Stream or the Agulhas Current. ${ }^{1-3,12-19}$ These vortices typically have a diameter of a few hundred kilometers, swirling velocities of $1-2 \mathrm{~m} / \mathrm{s}$, a rotation period of 2-10 days, a life span of several months to more than a year, and, whenever a reliable intrinsic drift estimate is possible, a westerly drift of several $\mathrm{cm} / \mathrm{s}$. When the rings have a cold (warm) core they circulate (anti)cyclonically and show large vertical deviations of the isopycnals (order of hundreds of meters) which makes that they present a positive (anticyclone) or negative (cyclone) mass anomaly with respect to their surroundings. The notion that a monopolar vortex carries a mass anomaly with respect to the fluid envelope in which they travel is central to the explanation of the intrinsic drift in terms of integral angular momentum in this paper.

The intrinsic drift of oceanic monopolar vortices has been studied in the context of various dynamical regimes and structures of background flows. ${ }^{20-27}$ These studies have in common that they represent the rotation of the solid earth by a $\beta$-plane, i.e., by an Euclidean plane endowed with a Coriolis force that increases with the "south-north" coordinate $y$. We return to the $\beta$-plane later in this section.

Theoretically a convenient definition of the drift velocity of a vortex on the $\beta$-plane is the velocity of the center of mass of the phenomenon. Let $U$ and $V$ be the components of this velocity along the standard Cartesian coordinates of the $\beta$-plane, respectively, $x$ ("west-east") and $y$ ("south-north").

In Ref. 25 a formula is derived for the drift velocity of shape-preserving geostrophic monopoles on a $\beta$-plane by expanding the shallow-water equations around a zeroth order geostrophic equilibrium on an f-plane. From the first order equation, including the $\beta$-effect, an integral formula, which unifies and extends results about intrinsic drift of the above cited studies, is given as

$$
U=-\frac{\beta_{0} \cdot a_{g}^{\prime}}{f^{2}} \frac{\int\left(H \cdot \eta+\frac{1}{2} \eta^{2}\right) d S}{\int \eta d S},
$$

$$
V=0 .
$$

In this equation, $H$ denotes the depth of the fluid far away from the vortex, where the fluid is motionless, while $\eta(x, y, t)$ is a dynamic field describing the deviation of depth from $H$ as function of space and time. The Coriolis parameter,

$$
f=2 \Omega \sin (\theta),
$$

where $\Omega$ is the angular frequency of the planet, quantifies the strength of the Coriolis force at lines $y=0$, while

$$
\beta_{0}=2 \frac{\Omega}{a} \cos (\theta)
$$

is a measure of the variation of $f(3)$ with the coordinate $y$, through $\theta: d y=a d \theta$, where $a$ represents the radius of the planet. Finally in expression (1) the symbol $a_{g}^{\prime}$ denotes the reduced counterpart of the acceleration of gravity $a_{g}$, 


$$
a_{g}^{\prime}=a_{g} \cdot \frac{\rho_{I I}-\rho_{I}}{\rho_{I I}} .
$$

The model is thus a reduced gravity single-layer model, consisting of a shallow layer of smaller density $\rho_{I}$ on top of a motionless lower layer of higher density $\rho_{I I}$.

Unfortunately the physical interpretation of intrinsic drift velocity (1), when derived from the shallow-water equations on a $\beta$-plane, is cumbersome as it involves the higher-order details of the vortex structure, in particular, its vorticity structure, rather than integral properties. In order to proceed, we therefore face some choices as to the dynamical variables and their conservation laws that we should use and to the geometric setting, i.e., the space, of the problem at hand. The basic choice of the principal dynamical variable is that between angular momentum, linear momentum, or quasigeostrophic potential vorticity. The basic choice as to the geometry is a spherical surface or the $\beta$-plane. The conservation law on the $\beta$-plane is principally the conservation of potential vorticity or the "relabeling symmetry" of the fluid, ${ }^{28}$ whereas angular momentum is not well defined on the $\beta$-plane. Moreover, a serious limitation, of both principal and practical relevance, that arises when a $\beta$-plane is chosen as the underlying space for an analysis of drift of vortices, is rooted in the limited spatial validity of the $\beta$-plane approximation. As was explicitly demonstrated for the momentum balance of a fluid in Ref. 29, in nonquasigeostrophic regimes, essential for strong vortex dynamics, the $\beta$-plane approximation is only locally a valid approximate setting for the dynamics along the surface of a rotating sphere. Because the drift of vortices in which we are interested has a global length scale, this is to be considered as a serious shortcoming of $\beta$-plane based analyses. This can be illustrated as follows. As was demonstrated in Ref. 29, for nonquasigeostrophic regimes, $\beta$-plane coordinates can be shown to be a valid local approximation of a locally geodesic spherical coordinate system on a rotating sphere. Hence north-south and west-east coordinate curves through the origin of the $\beta$-plane correspond to great circles on the approximated spherical surface. This implies that, if a westward drift of a vortex on a $\beta$-plane was to be interpreted on a sphere, one would either conclude that the vortex would proceed along a great circle-which conclusion, as we see in the present paper, is erroneous—or one would argue that, due to the limited spatial validity of the $\beta$-plane approximation, no conclusion about the global trajectory on the sphere can be derived other than the conclusion that the vortex leaves the origin in a westwardly direction. This later conclusion is a valid but dissatisfying result for any study that aims to provide understanding of trajectories of vortices. On the sphere, however, apart from the conservation of potential vorticity and its related relabeling symmetry, also the angular momentum and its relationship with rotational symmetry are well defined. Moreover, on a sphere the vorticity is related to the spatial derivative of the angular momentum ${ }^{30}$ or, vice versa, the angular momentum is an integral property of the vorticity field. Since we aim at relating the intrinsic drift to integral properties of the principal dynamical variable, it seems logical to make a choice for the combination of angular momentum dynamics on a spherical surface. Interestingly this is also the approach in Ref. 31, where the angular momentum dynamics of a spinning solid disk on a rotating sphere is discussed. As a model of fluid vortices, however, the solid disk does not suffice: its drifting sense, westward or eastward, depends on its spinning sense, whereas the intrinsic drift of all monopolar vortices is retrograde (westward), irrespective of spin orientation.

In the present paper we explicitly demonstrate that the retrograde intrinsic drift of monopolar vortices may be clearly understood in terms of the evolution of the integral angular momentum of a reduced gravity shallow-water model, carrying a vortex, on a rotating sphere. The shortcomings and difficulties discussed above are overcome along these lines.

The structure of the paper is then as follows. In Sec. II we introduce a suitable formulation of the covariant momentum equation for a fluid. From this, referring to vector fields that generate rotations, which are the principal geometrical transformations behind angular momentum dynamics, we derive the covariant angular momentum equation for a geophysical fluid in Sec. III and the implied integral angular momentum equation for a fluid shell on a rotating sphere in Sec. IV.

To apply this dynamics to vortices we use suitable representations on a spherical surface of vector fields that generate rotations (Appendix D), a coordinate system that can be attached to a 
vortex and move along with it (Appendix E), and local approximations of several fields with respect to this coordinate system (Appendix F). To prevent the development of these tools from obscuring the main line of reasoning, we develop the tools in Appendices D and F. In Sec. V then we introduce a suitable way to distinguish a vortex from its background and formulate the angular momentum dynamics of it.

So far the dynamics is studied in a coordinate-free formalism. In Sec. VI we develop a concrete representation of the angular momentum equations with respect to a coordinate system that is suitable for localized vortices ${ }^{26}$ that move along the surface of the planet and study these concrete equations numerically in Sec. VII and analytically in Sec. VIII. In Sec. IX we summarize the results and represent them in terms of angular momentum vector diagrams.

\section{COVARIANT FORMULATION OF MOMENTUM BALANCE OF FLUID ELEMENTS}

The momentum equation for a fluid with respect to a coordinate system, the rotation of which is described by a time-dependent angular velocity pseudovector $\vec{\Sigma}(t)$, is in vector notation, ${ }^{32}$

$$
\frac{d \vec{v}}{d t}+\vec{v} \cdot \nabla \vec{v}+\frac{d \vec{\Sigma}}{d t} \times \vec{r}+2 \vec{\Sigma} \times \vec{v}-\frac{1}{2} \nabla\left(|\vec{\Sigma} \times \vec{r}|^{2}\right)=-\nabla \phi+\frac{1}{\rho} \nabla \cdot \vec{\tau} .
$$

Here $\vec{v}$ denotes the velocity field of the fluid, $\vec{r}$ is the position vector with respect to the origin, and $\overrightarrow{\vec{\tau}}$ is the stress tensor. In this study $\phi$ denotes the gravitational potential of the planet; ${ }^{33}$ the details of the structure of $\phi$, as relevant to the present paper, are discussed in Appendix F 2 a.

The following vector field gives the velocity of the rotating coordinate system with respect to an inertial coordinate system:

$$
\vec{s}(\vec{r}, t)=\vec{\Sigma}(t) \times \vec{r}
$$

note that the field $\vec{s}$ is position dependent in contrast to $\vec{\Sigma}$ which is homogeneous in space at each fixed moment in time.

Equation (6) may be rewritten in terms of the field $\vec{s}$; we do so and adopt covariant notation at the same time,

$$
\frac{\delta v_{p}}{\delta t}+\frac{\partial s_{p}}{\partial t}+d s_{j p} v^{j}-\frac{1}{2} \nabla_{p}\left(s_{q} s^{q}\right)=-\nabla_{p} \phi+\frac{1}{\rho} \nabla_{j} \tau_{p}^{j} .
$$

The first term of this equation is expressed in terms of the differential operator,

$$
\frac{\delta v_{p}}{\delta t}=\frac{\partial v_{p}}{\partial t}+v^{q} \nabla_{q} v_{p}
$$

where $\nabla_{q} v_{p}$ denotes the covariant derivative, which in turn may be expressed in terms of Christoffel symbols $\Gamma_{j k}^{i},{ }^{34}$ as

$$
\nabla_{q} v_{p}=\frac{\partial}{\partial x^{q}} v_{p}-\Gamma_{q p}^{r} v_{r}
$$

expressions for Christoffel symbols with respect to a spherical coordinate system are tabulated in Table IV.

Because the exterior derivative, $d s_{j p}=\left(\partial / \partial x^{j}\right) s_{p}-\left(\partial / \partial x^{p}\right) s_{j}$, describes the Coriolis effect, we refer to $d s_{j p}$ as to the Coriolis tensor. For $v_{p}=-s_{p}$, which refers to a fluid at rest in an inertial frame, (8) reduces to the hydrostatic equation. 


\section{COVARIANT FORMULATION OF ANGULAR MOMENTUM BALANCE}

\section{A. Rotation and angular momentum}

In this section we derive from (8) the covariant angular momentum equation for a non-selfgravitating spherical fluid shell corotating with the gravitating solid core of a celestial body; briefly "the rotating system." The fluid shell itself is thought to be a reduced gravity single-layer, shallow-water model as discussed in Sec. I.

Because, as we see, both the gravitational potential and the solid body break the spherical symmetry of the system, not all components of angular momentum, neither at fluid element level nor at integral angular momentum level, are conserved. For that reason application of Noether's theorem, ${ }^{35}$ which relates symmetries of dynamical systems to conservation laws, cannot reveal the full vector equation governing angular momentum dynamics of this system. However, generalizing Noether's theorem, neglecting friction, in order to become able to study the consequences of broken symmetries, Van der Toorn ${ }^{36}$ derived the full angular momentum equations for shallow water on a rotating planet from the broken spherical symmetry of the system.

In this paper we present a shorter and more direct derivation of these angular momentum equations as follows. In Sec. III B by straightforward tensor analysis we derive from (8) a general identity for the product of the velocity of fluid elements with an arbitrary vector field. This identity already presents the basic form of the angular momentum equation. The derivation is interesting in that it shows that the Coriolis tensor, which in the momentum balance plays such a prominent role, is decomposed here and really distributed over two seemingly unrelated tensors, namely, a material time derivative and a Lie derivative of the velocity of the coordinate system. This shows why, in the context of angular momentum dynamics, the Coriolis force does not play such a prominent role as it does in momentum dynamics.

In Sec. III D we show how the general identity drastically simplifies - a nonlinear term cancels - thanks to a property of vector fields that generate rotations, presented in Sec. III C in an ad hoc manner. Thus, we show in a direct way that angular momentum obeys relatively simple dynamics as a consequence of its close relationship to rotations.

\section{B. The inner product of the momentum balance with an arbitrary vector field}

It is shown in Appendix A that the inner product of momentum equation (8) and an arbitrary vector field $\Psi$ gives

$$
\rho\left(\frac{d}{d t}\left(\Psi^{k} \hat{v}_{k}\right)-v^{k} \mathcal{L}_{\Psi}(s)_{k}+\Psi^{k} \nabla_{k} \Upsilon-\hat{v}_{k} \frac{\partial}{\partial t} \Psi^{k}\right)=\rho v^{p} v^{q} \nabla_{q} \Psi_{p}+\Psi^{k} \nabla_{j} \tau_{k}^{j},
$$

in this expression, and in the rest of this paper, we employ the Einstein summation convention, i.e., we sum over repeated indices. The tensor $\mathcal{L}_{\Psi}(s)_{q}$, defined as

$$
\mathcal{L}_{\Psi}(s)_{q}=s_{p} \nabla_{q} \Psi^{p}+\Psi^{p} \nabla_{p} s_{q},
$$

is known as the Lie derivative of the covector field $s_{q}$ along the vector field $\Psi^{p}$; furthermore,

$$
Y=\phi-\frac{1}{2} s_{k} s^{k} \quad \text { and } \quad \hat{v}=v+s .
$$

In terms of the conventional vector notation of Eq. (7) the general potential $Y$ is

$$
Y=\phi-\frac{1}{2}\left(|\vec{\Sigma} \times \vec{r}|^{2}\right)
$$

it is the sum of the gravitational potential $\phi$ and a general centrifugal potential, $\phi_{s}=-\frac{1}{2}\langle s, s\rangle[\mathrm{see}$ (8)], associated with the absolute velocity $s$ of the coordinate system. It is further discussed in Appendix F 2 a. 


\section{Vector fields that generate rotations}

Because angular momentum is associated with rotations, we introduce the following vector fields that generate rotations, represented here with respect to Cartesian coordinates $c^{1}, c^{2}$, and $c^{3}$ :

$$
\begin{aligned}
& \mathbf{r}_{1}=\left(0,-c^{3}, c^{2}\right), \\
& \mathbf{r}_{2}=\left(c^{3}, 0,-c^{1}\right), \\
& \mathbf{r}_{3}=\left(-c^{2}, c^{1}, 0\right) .
\end{aligned}
$$

Flows along the fields, $\mathbf{r}_{1}, \mathbf{r}_{2}$, and $\mathbf{r}_{3}$, correspond to rotations about the Cartesian, $c^{1}-, c^{2}$ - and $c^{3}$-axes, respectively. Therefore, these fields are generators of rotations. If $\left(\boldsymbol{\Phi}^{1}, \boldsymbol{\Phi}^{2}, \boldsymbol{\Phi}^{3}\right)$ denotes a Cartesian vector, then the following vector field $\Phi$ is a generator of rotations about the vector $\left(\boldsymbol{\Phi}^{1}, \boldsymbol{\Phi}^{2}, \boldsymbol{\Phi}^{3}\right)$ :

$$
\left.\Phi=\boldsymbol{\Phi}^{\mu} \mathbf{r}_{\mu} \quad \text { (sum over } \mu \in\{1,2,3\}\right) .
$$

The Lie bracket $[\cdot, \cdot]$, which is a special case of the Lie derivative,

$$
[u, v]=\mathcal{L}_{u} v=u^{j} \frac{\partial}{\partial x^{j}} v^{i}-v^{j} \frac{\partial}{\partial x^{j}} u^{i},
$$

forms a product on the three-dimensional vector space which contains all linear combinations of $\mathbf{r}_{1}, \mathbf{r}_{2}$, and $\mathbf{r}_{3}$. This vector space endowed with this product forms a Lie algebra. The product table can be summarized as

$$
\left[\mathbf{r}_{\mu}, \mathbf{r}_{\nu}\right]=-\epsilon_{\mu \nu}{ }^{\lambda} \mathbf{r}_{\lambda},
$$

where $\mu, \nu, \lambda \in\{1,2,3\}$ and the symbol $\epsilon_{\mu \nu}{ }^{\lambda}$ equals 1 if $(\mu, \nu, \lambda)$ forms an even permutation of (1, $2,3),-1$ if it forms an odd permutation and equals 0 if otherwise. Multiplication table (17) shows that $\mathbf{r}_{1}, \mathbf{r}_{2}$, and $\mathbf{r}_{3}$ span the so(3) algebra of rotations.

The covariant derivative of an arbitrary element of so(3) plays a major role in our construction of the angular momentum equation for continua. On the basis of (14), it is easily checked that in Cartesian coordinates $\nabla_{i}\left(\mathbf{r}_{\mu}\right)_{j}=-\nabla_{j}\left(\mathbf{r}_{\mu}\right)_{i}$ for all $\mu \in\{1,2,3\}$. Because skew symmetry of a tensor is a coordinate-free property and because each linear combination of skew-symmetric tensors is itself skew symmetric, we have the general property,

$$
\nabla_{i} \Phi_{j}=-\nabla_{j} \Phi_{i} \text { for all } \Phi \in \mathrm{so}(3) .
$$

\section{Focusing on generators of rotations}

All terms of (11) are linear in the velocity field $v$ with exception of the first term on the right hand side. If we choose $\Psi$ from so(3), however, then according to (18) the tensor $\nabla_{q} \Psi_{p}$ is skew symmetric and, therefore,

$$
\Phi \in \operatorname{so}(3) \Rightarrow v^{p} v^{q} \nabla_{q} \Phi_{p}=0 .
$$

We substitute such a $\Phi$ for $\Psi$ in (11) to find

$$
\rho \frac{d}{d t}\left(\Phi^{k} \hat{v}_{k}\right)=\rho v^{k} \mathcal{L}_{\Phi}(s)_{k}-\rho \Phi^{k} \nabla_{k} \Upsilon+\rho \hat{v}_{k} \frac{\partial}{\partial t} \Phi^{k}+\Phi^{k} \nabla_{j} \tau_{k}^{j} .
$$

In an inertial system, i.e., for $s=0$, represented with respect to Cartesian coordinates and with stationary $\Phi$, this equation is easily recognized as an angular momentum equation.

Equation (20) is a covariant formulation of angular momentum balance of fluid elements with respect to an arbitrarily moving coordinate system. The equation holds for any choice of vector 
field $\Phi$ from so(3). Since so(3) is a three-dimensional linear space of vector fields, we can choose three linearly independent vector fields for $\Phi$ so that (20) should be understood as a set of three independent equations. If we choose the fields, $\mathbf{r}_{1}, \mathbf{r}_{2}$, and $\mathbf{r}_{3}$, from (14), for example, we find three equations for $\mathbf{r}_{1}{ }^{k} \hat{v}_{k}, \mathbf{r}_{2}{ }^{k} \hat{v}_{k}$, and $\mathbf{r}_{3}{ }^{k} \hat{v}_{k}$, respectively. Because these three quantities are inner products of vector fields, they are scalar objects in sharp contrast to the more classical form of angularmomentum $\vec{r} \times \vec{v}$, which is a pseudo vector. (Müller ${ }^{30}$ studied covariant formulations of geophysical angular momentum budgets based on the form $\vec{r} \times \vec{v}$.) The relation between the scalar fields and this classical form of angular momentum is

$$
\begin{aligned}
& \mathbf{r}_{1}{ }^{k} \hat{v}_{k}=\vec{e}_{1} \cdot \vec{r} \times \vec{v}, \\
& \mathbf{r}_{2}{ }^{k} \hat{v}_{k}=\vec{e}_{2} \cdot \vec{r} \times \vec{v}, \\
& \mathbf{r}_{3}{ }^{k} \hat{v}_{k}=\vec{e}_{3} \cdot \vec{r} \times \vec{v},
\end{aligned}
$$

where the $\vec{e}_{n}$ are the unit vectors of the Cartesian coordinate system $\left(c^{1}, c^{2}, c^{3}\right)$ of (14). In a Cartesian representation the left hand sides of Eqs. (21) correspond just to the components of $\vec{r}$ $\times \vec{v}$, but in other curvilinear coordinate representations this is not the case: one should take the inner product of the curvilinear representation of $\vec{r} \times \vec{v}$ and the curvilinear representation of the unit vector fields $\vec{e}_{n}$ of the Cartesian coordinate system to construct curvilinear representations of the central quantities $\Phi^{k} \hat{v}_{k}$ of (20).

In this paper we follow the more direct approach suggested by the tensorial form of (20) and (in Appendices D and E) construct suitable spherical representations of the generators of rotations, i.e., of so(3), and thus come to a suitable spherical coordinate representation of the angular momentum balance.

\section{APPLICATION TO A REDUCED GRAVITY, SINGLE-LAYER MODEL ON A SPHERE}

\section{A. Independence of the radial coordinate}

In this section we derive the integral covariant angular momentum equation for the so-called reduced gravity single-layer model, on a rotating solid sphere.

A "reduced gravity single-layer model" refers to the outer layer of a system that consists of two shells of fluid that are both thin in the sense that their depths are much smaller than the radius of the planet. Moreover, the depth of the outer layer is thin compared to that of the inner layer and has a lower density. The inner shell of fluid covers the solid sphere and is supposed to be passive, which means that it does not carry a tangential pressure gradient. The outer shell of fluid covers the inner shell. The outer shell is allowed to show dynamic behavior, and we formulate an integral angular momentum equation for the outer shell.

In the context of a spherical system we employ spherical coordinates. With respect to such coordinates, the third, radial, component of the vector field $\Phi$ vanishes, as does the third component of the field $s$. Furthermore, the contravariant components of $\Phi$ and $s$ do not depend on the radial coordinate: the first and second contravariant components in spherical coordinates represent angular velocities and these do not depend on the distance to the center for solid rotations about the center.

Because the fluid shells are shallow compared to the planet's radius, we may approximate, in the metric, the value of the radial coordinate by the value of the planet's radius. In such an approximation the covariant components of $\Phi$ and $s$ then also do not depend on the radial coordinate. We then have

$$
\mathcal{L}_{\Phi}(s)_{3}=0
$$

The sum over the index $k$ in (20) gives therefore no contribution for $k=3$. 


\section{B. Integral angular momentum of the outer shell}

Let $V$ be the volume of the outer fluid shell. We study the integral of (20) over the volume $V$. Because the whole volume $V$ is a material volume, we may apply the transport theorem for material derivatives, ${ }^{37}$

$$
\int_{V} \rho_{I} \frac{d}{d t}\left(\Phi^{k} \hat{v}_{k}\right) d V=\frac{d}{d t} \int_{V} \rho_{I}\left(\Phi^{k} \hat{v}_{k}\right) d V
$$

If we, furthermore, assume that $v$ does not depend on the radial coordinate, we may integrate the right hand side and find in terms of the depth $H(1+h(\lambda, \theta, t))$ that

$$
\int_{V} \rho_{I} \frac{d}{d t}\left(\Phi^{k} \hat{v}_{k}\right) d V=\frac{d}{d t} \int_{S} \rho_{I}\left(\Phi^{k} \hat{v}_{k}\right) H(1+h) d S
$$

$H$ is the constant order of magnitude and $h(\lambda, \theta, t)$ the modulation of the depth as a function of geographical longitude $\lambda$, latitude $\theta$, and time $t$. The integral over $S$ denotes integration over the entire range of longitude and latitude.

\section{Integral angular momentum equation for a reduced gravity, shallow-water model on a sphere}

In Appendices B and C we show that the stress tensor only exerts a net integral torque through the boundaries of the fluid and that pressure does not contribute to the integral angular momentum balance when the bottom of the fluid layer coincides with a surface of constant geopotential; i.e., the bottom is "flat."

When we neglect friction, stress reduces to pressure force and hence no integral torque can result from stress. Then we can derive from (20), on basis of the observations in Secs. IV A-IV C, that within a reduced gravity single-layer model for a fluid layer on a rotating sphere it holds $(\bar{n} \in\{1,2\})$ that

$$
\frac{d}{d t} \int_{S}\left(\Phi^{\bar{n}} \hat{v}_{\bar{n}}\right)(1+h) d S=\int_{S}\left(v^{\bar{n}} \mathcal{L}_{\Phi}(s)_{\bar{n}}-\Phi^{\bar{n}} \nabla_{\bar{n}} \Upsilon+\hat{v}_{\bar{n}} \frac{\partial}{\partial t} \Phi^{\bar{n}}\right)(1+h) d S .
$$

This equation is essentially a two-dimensional equation in the sense that it depends only on components tangential to the sphere of all vector fields involved.

In terms of the inner product of vector fields (D11), that is, introduced in Appendix D 2, Eq. (25) may be expressed in a natural way,

$$
\frac{d}{d t}\langle\Phi \| \rho(s+v)\rangle=\langle[\Phi, s] \| \rho v\rangle-\langle\Phi \| \rho d \Upsilon\rangle+\left\langle\frac{\partial}{\partial t} \Phi \| \rho(v+s)\right\rangle .
$$

In the first term on the right hand side we have used bracket notation (16) for Lie derivatives. Furthermore, in (26) we use abbreviations (13) and

$$
\rho(\lambda, \theta, t) \equiv 1+h(\lambda, \theta, t),
$$

which represents a dimensionless density (mass per surface element).

As long as we refrain from employing coordinate systems that deform as a function of time, i.e., as long as the velocity field of the coordinate system $s$, is a Killing field [a field $s$ such that $\mathcal{L}_{s} g_{i j}=0$, which is true on a spherical surface if and only if $\left.s \in \operatorname{so}(3)\right]$, the metric does not depend on $t$. Then Eq. (26) reduces to

$$
\left\langle\Phi \| \frac{d}{d t} \rho(s+v)\right\rangle+\langle\Phi \| \rho d \Upsilon\rangle=\langle[\Phi, s] \| \rho v\rangle .
$$

We refer to (28) as to the general angular momentum equation. 


\section{DISTINCTION BETWEEN PHENOMENON AND BACKGROUND MASS CURRENTS}

\section{A. Decomposition of mass currents}

We assume that we have a coordinate system which is attached to the center of mass of a monopolar vortex. Thus, with respect to such a coordinate system, the monopole does not drift.

Let the velocity of the coordinate system with respect to the planet be described by a vector field $\nu \in \operatorname{so}(3)$. Then, if $\omega$ denotes the velocity of the planet with respect to inertial space, the motion of the coordinate system with respect to an inertial system of reference is

$$
s=\omega+\nu .
$$

We assume that, apart from motions associated with the vortex, the fluid is at rest with respect to the planet. This implies that, as seen from our coordinate system, the fluid background stays behind, i.e., moves with velocity $-\nu$ with respect to our coordinate system.

In the light of (27) we should associate a mass density $\rho=1$ with the background if we describe the background's dimensionless depth by $h(\lambda, \theta, t)=0$. Therefore, a dimensionless mass current equal to $-\nu$ is associated with the background.

Note the above involves the approximation that at equilibrium the surfaces of the outer and inner fluid layers of the reduced gravity single-layer model are spherical surfaces. In other words all surfaces of constant geopotential, as seen in a reference system that is corotating with the solid body, are approximated by spherical surfaces. This approximation has been recently reviewed and justified in Ref. 33.

We now decompose the total mass current in a vortex-associated part $\rho \breve{v}$ and a background part $-\nu$,

$$
\rho v=\rho \breve{v}-\nu .
$$

This relation can also be interpreted as the definition of the velocity field $\breve{v}$ that we associate with the vortex.

\section{B. The angular momentum equation for a phenomenon}

Due to the linearity of general angular momentum equation (28) in the mass currents, the angular momentum equation can be decomposed as follows.

Substituting the relation of total and phenomenon-associated mass currents (30) into the general angular momentum equation (28), we find

$$
\left\langle\Phi \| \frac{d}{d t}(\rho \breve{v}-\nu+\rho s)+\rho d \Upsilon\right\rangle=\langle[\Phi, s] \| \rho \breve{v}-\nu\rangle .
$$

According to (29) and (27) and due to the linearity of the inner product in its arguments, Eq. (31) may be expanded as follows:

$$
\left\langle\Phi \| \frac{d}{d t}(\omega)+d \Upsilon\right\rangle+\left\langle\Phi \| \frac{d}{d t}(\rho \breve{v}+h s)+h d \Upsilon\right\rangle=-\langle[\Phi, s] \| \nu\rangle+\langle[\Phi, s] \| \rho \breve{v}\rangle .
$$

The first terms on the left- and right-hand sides of this equation turn out to form a subidentity, i.e.,

$$
\left\langle\Phi \| \frac{d}{d t} \omega\right\rangle=\langle\Phi \|-d \Upsilon\rangle-\langle[\Phi, s] \| \nu\rangle
$$

holds. That this relation holds, in general, can be seen as follows. On the basis of the reasoning given after (C3) we conclude that the first term on the right hand side vanishes, 


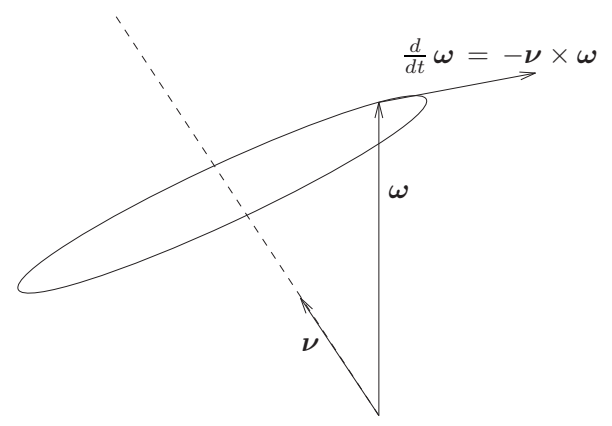

FIG. 1. Diagram of relation (38): the coordinate system rotates with respect to the planet about the vector $\boldsymbol{\nu}$. As a result, the vector $\boldsymbol{\omega}$, which is steady with respect to the planet, is observed to trace a circular path with respect to the coordinate system.

$$
\langle\Phi \|-d Y\rangle=0
$$

This expression may be read as the statement that the potential $Y$ cannot exert a torque on the background, which has dimensionless density of 1 , as a whole.

When we use (34), relation (29), and the skew symmetry of the nested bracket $\langle[\cdot, \cdot] \| \cdot\rangle$ with respect to elements of so(3) (see Appendix D 3), Eq. (33) may be written as

$$
\left\langle\Phi \| \frac{d}{d t} \omega\right\rangle=\langle\Phi \|[\nu, \omega]\rangle .
$$

The validity of this equation follows immediately from the identity

$$
\frac{d}{d t} \omega=[\nu, \omega]
$$

It can be demonstrated that this relation holds for any pair of smooth vector fields, $\omega$ and $\nu$, if $\nu$ denotes the velocity of the coordinate system and on the condition that $d \omega / d t$ vanishes if $\nu$ vanishes; but here, using the fact that $\omega, \nu \in \operatorname{so}(3)$, we can give a graphical explanation of identity (36). We expand $\omega$ and $\nu$ with respect to the basis $\mathbf{r}_{1}, \mathbf{r}_{2}$, and $\mathbf{r}_{3}$ of so(3),

$$
\omega=\omega^{\mu} \mathbf{r}_{\mu}, \quad \nu=\nu^{\mu} \mathbf{r}_{\mu} .
$$

With (17) it then readily follows that the coefficients $\boldsymbol{\omega}$ and $\boldsymbol{\nu}$ obey the Cartesian relation

$$
\frac{d}{d t} \boldsymbol{\omega}=-\boldsymbol{\nu} \times \boldsymbol{\omega},
$$

that shows that the vector $\boldsymbol{\omega}$, which points along the axis of rotation of the planet and has length $\|\boldsymbol{\omega}\|=\Omega$ and which is thus steady with respect to the planet, may be moving with respect to the coordinate system just because the coordinate system rotates about the axial vector $\boldsymbol{\nu}$ with angular frequency $\|\boldsymbol{\nu}\|$; see Fig. 1.

In the light of relation (33), Eq. (32) reduces to

$$
\left\langle\Phi \| \frac{d}{d t}(h s+\rho \breve{v})+h d \Upsilon\right\rangle=\langle[\Phi, s] \| \rho \breve{v}\rangle .
$$

\section{CONCRETE REPRESENTATION SUITABLE FOR LOCALIZED PHENOMENA}

To apply angular momentum (39) to localized phenomena such as monopolar vortices, we represent it by a set of three differential equations, one for each independent component of the 
TABLE I. Local inner products are encountered during the evaluation of Eq. (40).

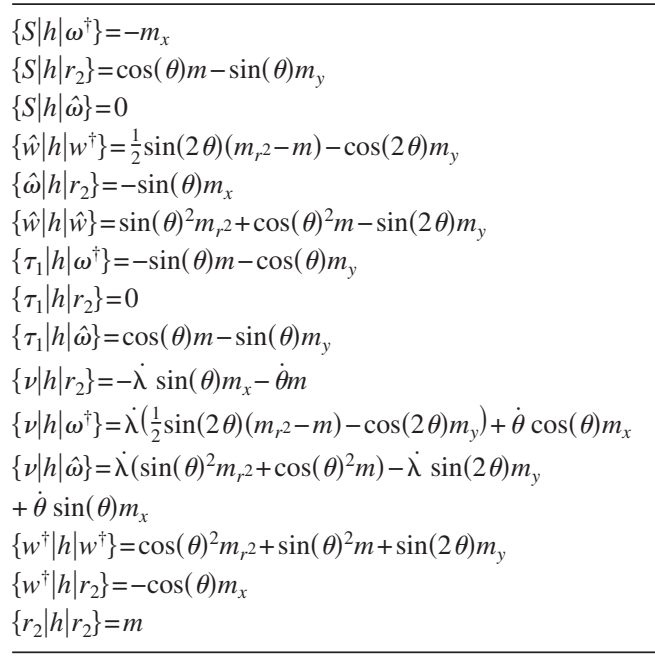

integral angular momentum. Furthermore, we exploit the localized character of the phenomena and approximate spherical representations by local Cartesian ones wherever possible.

In order to formulate adequate differential equations we start by noting that, as far as the argument $\Phi$ is concerned, (39) is of the form $A(\Phi)=B(\Phi), \forall \Phi \in \operatorname{so}(3)$, where $A, B: \operatorname{so}(3) \rightarrow \mathbb{R}$, are linear maps. From this linearity in $\Phi$ it follows that, if Eq. (39) holds for three independent basis elements of so(3), then it holds for all elements of so(3). Therefore, (39) is equivalent to three equations that can be constructed by substituting the elements of a basis for so(3). Several such bases, to be formed by three vector fields on the spherical surface, each being a generator of a rotation, are developed in Appendices $\mathrm{D}$ and $\mathrm{E}$. Because of its physical content we use the basis $\left(\omega^{\dagger}, r_{2}, \hat{\omega}\right)$; see expressions (E12), (D8), (E7), and (E11). If the phenomenon under study, e.g., a vortex, is sufficiently localized, i.e., if the fields $h$ and $\breve{v}$ vanish sufficiently quickly in regions away from the origin, then the inner products in (39) can be approximated locally. The integral over all of the sphere is then replaced by an integral in the local Euclidean neighborhood of the phenomenon which can be described in terms of Cartesian coordinates. A suitable local inner product $\{\cdot \| \cdot\}$ (F13) is discussed in Appendix F 3,

$$
\left\{\Phi \| \frac{d}{d t}(h s+\rho \breve{v})+h d \Upsilon\right\}=\{[\Phi, s] \| \rho \breve{v}\} .
$$

Like (39), (40) is linear in $\Phi$ so that, as explained above, it can be represented by the three differential equations that follow when we subsequently substitute $\Phi=\hat{\omega}, \Phi=\omega^{\dagger}$, and $\Phi=r_{2}$. To represent the velocity of the coordinate system $s$ we use $s=\Omega \hat{\omega}+\nu$ [see (29) and (E9)].

In the local inner products local approximations of the arguments may be used with one exception: the commutators $[\cdot, \cdot \cdot]$ within the local inner products should be evaluated using global vector fields. This is because, as is discussed in Appendix F 1, the proper Lie algebra so(3), which rules angular momentum dynamics, cannot be produced by the local approximations of the generators of rotations. Therefore, to evaluate $[\Phi, s]$ we use the commutators (E14)-(E17) presented in Appendix E 3.

The terms of Eqs. (40) can be evaluated approximately, using local approximations of the metric (Appendix F 3), gravitational potential (F9), and the vector fields $\hat{\omega}, \omega^{\dagger}$, and $r_{2}[(\mathrm{~F} 2)-(\mathrm{F} 4)]$. Local inner products that are encountered during the evaluation of (40) are listed in Table I. These inner products are expressed in terms of the total mass anomaly $m=\int h d x d y$ and the moments $m_{x}=\int x h d x d y, m_{y}=\int y h d x d y$, and $m_{r^{2}}=\int\left(x^{2}+y^{2}\right) h d x d y$.

By means of the condition, 


$$
m_{x}=0 \text { and } m_{y}=0,
$$

we have fixed the center of mass at the origin of our coordinate system.

On the basis of this all we arrive at the following set of equations:

$$
\begin{gathered}
\ddot{\lambda}\left(\cos (\theta)^{2} m+\sin (\theta)^{2} m_{r^{2}}\right)+\dot{\theta}\left(-\sin (2 \theta)(\Omega+\dot{\lambda})\left(m-\frac{1}{2} m_{r^{2}}\right)+\cos (\theta)\left\{\tau_{0} \| \rho \breve{v}\right\}\right) \\
+(\Omega+\dot{\lambda}) \sin (\theta)^{2} \frac{d m_{r^{2}}}{d t}+\sin (\theta) \frac{d}{d t}\left\{\tau_{0} \| \rho \breve{v}\right\}=0, \\
\ddot{\lambda}\left(\frac{1}{2} \sin (2 \theta)\left(m_{r^{2}}-m\right)\right)+\dot{\theta}\left[(\Omega+\dot{\lambda})\left(2 \sin (\theta)^{2} m+\cos (\theta)^{2} m_{r^{2}}\right)-\sin (\theta)\left\{\tau_{0} \| \rho \breve{v}\right\}\right] \\
+\frac{1}{2}(\Omega+\dot{\lambda}) \sin (2 \theta) \frac{d m_{r^{2}}}{d t}+\cos (\theta) \frac{d}{d t}\left\{\tau_{0} \| \rho \breve{v}\right\}=0, \\
m \ddot{\theta}+\frac{1}{2} m \sin (2 \theta) \dot{\lambda}(\dot{\lambda}+2 \Omega)=(\Omega+\dot{\lambda}) \cos (\theta)\left\{\tau_{0} \| \rho \breve{v}\right\} .
\end{gathered}
$$

The field $\tau_{0}$ (F5) is the generator of rotations about the origin of the local coordinate system that is fixed at the center of the vortex. The bracket $\left\{\tau_{0} \| \rho \breve{v}\right\}$ may therefore be interpreted as a measure of the total monopolar vortex strength of the field $\rho \breve{v}$.

Now we scale the dimensional time $t^{*}$ as $t^{*}=t / \Omega$ and write derivatives of $\lambda$, $\theta$, etc., with respect to $t^{*}$ and $t$, respectively, as $\dot{\lambda}$ and $\lambda^{\prime}$ so that

$$
\dot{\lambda}=\Omega \lambda^{\prime}, \quad \dot{\theta}=\Omega \theta^{\prime}, \quad \text { etc. }
$$

Furthermore, we introduce

$$
q=m_{r^{2}} / m
$$

and

$$
\varpi=\frac{\left\{\tau_{0} \| \rho \breve{v}\right\}}{m \cdot \Omega} .
$$

This leads to the following scaled form of Eqs. (42)-(44):

$$
\begin{gathered}
\left(\cos (\theta)^{2}+q \sin (\theta)^{2}\right) \lambda^{\prime \prime}+\sin (\theta)^{2} q^{\prime}\left(1+\lambda^{\prime}\right)+\sin (\theta) \varpi^{\prime} \\
+\theta^{\prime}\left(\cos (\theta) \varpi-\left(1-\frac{q}{2}\right) \sin (2 \theta)\left(1+\lambda^{\prime}\right)\right)=0, \\
\frac{1}{2}(q-1) \sin (2 \theta) \lambda^{\prime \prime}+\frac{1}{2} \sin (2 \theta) q^{\prime}\left(1+\lambda^{\prime}\right)+\cos (\theta) \varpi^{\prime} \\
+\theta^{\prime}\left(\left(\cos (\theta)^{2} q+2 \sin (\theta)^{2}\right)\left(1+\lambda^{\prime}\right)-\sin (\theta) \varpi\right)=0, \\
\theta^{\prime \prime}-\cos (\theta) \varpi\left(1+\lambda^{\prime}\right)+\frac{1}{2} \sin (2 \theta) \lambda^{\prime}\left(2+\lambda^{\prime}\right)=0 .
\end{gathered}
$$

\section{A. Sign of dimensionless vortex strength}

The sign of the dimensionless vortex strength $\varpi$ depends on the hemisphere in which the vortex finds itself and on the orientation of the vortex, i.e., on whether it is cyclonic or anti cyclonic. 
TABLE II. Turning senses (top views and signs), signs of mass anomalies $m$, and the resulting signs of the ratio of these quantities, for cyclones $(C)$ and regular $\left(A_{r}\right)$ and irregular $\left(A_{i}\right)$ anticyclones on the northern and southern hemispheres.

\begin{tabular}{|c|c|c|c|c|c|c|}
\hline & \multicolumn{3}{|c|}{ Northern h.s. } & \multicolumn{3}{|c|}{ Southern h.s. } \\
\hline & $\mathrm{C}$ & $\mathrm{A}_{\mathrm{r}}$ & $\mathrm{A}_{\mathrm{i}}$ & $\mathrm{C}$ & $\mathrm{A}_{\mathrm{r}}$ & $\mathrm{A}_{\mathrm{i}}$ \\
\hline Appearance & $a$ & $\diamond$ & ๑ & $\diamond$ & a & Q \\
\hline$\left\{\tau_{0} \| \rho \breve{v}\right\}$ & + & - & - & - & + & + \\
\hline$m$ & - & + & - & - & + & - \\
\hline$\varpi$ & - & - & + & + & + & - \\
\hline
\end{tabular}

This is demonstrated in detail in Table II. The table is divided into two main columns, one for each hemisphere. The first row of the table depicts, schematically, the top view of a particular type of vortex. The first column, for example, shows a cyclone on the Northern hemisphere. Because it has the same orientation as the field $\tau_{0}$ the bracket $\left\{\tau_{0} \| \rho \breve{v}\right\}$ is of positive sign, as is indicated in the second row of Table II.

Cyclones always have negative mass (and pressure) anomalies: both the Coriolis- and the centrifugal-forces point outward in a cyclone so they can only be balanced by an inward pressure force. The center of the cyclone must therefore be a region of low pressure and thus of negative mass anomaly; the sign of the mass anomaly is indicated in the third row.

In anticyclones horizontal components of Coriolis forces are inward while centrifugal forces, associated with the swirling motion of the fluid inside the vortex, are outward. Therefore, of anticyclones we distinguish two types as follows.

Regular anticyclones are anticyclones in which the horizontal component of the Coriolis force dominates the centrifugal force. The sum of Coriolis and centrifugal force is therefore inward within the kernel of the vortex and this total force must therefore be balanced by an outward pressure force. As a result these vortices have positive mass anomaly.

By their definition it follows that regular anticyclones cannot exist at the equator for there the horizontal component of the Coriolis force vanishes.

In irregular anticyclones the centrifugal force is dominant and the mass anomaly is negative. According to Ref. 1 irregular anticyclones "are seen in the geophysical context (for example, eddies with low-pressure centers that are carried across the equator to form anomalous lows)." Anticyclonic oceanic rings, however, are always regular. ${ }^{1}$

As is shown by the last row of Table II, for regular monopoles the sign of $\varpi$ depends exclusively on the hemisphere: it is negative on the northern and positive on the southern hemisphere.

\section{NUMERICAL SOLUTIONS}

\section{A. Closing the angular momentum equations: The Gaussian monopole}

In the present section, by presenting various examples of numerical solutions of Eqs. (48)-(50), we give an overview of qualitative types of vortex behavior covered by these equations and show how these depend on vortex type.

Although in later sections we shall see that the results to be presented here can be understood and reproduced analytically from Eqs. (48)-(50) as they are, one should realize that these equations do not constitute a closed set of dynamical equations for they consist of three equations describing the relation of four dynamical variables, namely, the positional coordinates of the vortex $\lambda$ and $\theta$, its spin $\left\{\tau_{0} \| \rho \breve{v}\right\}$, as well as its moment of inertia $m_{r^{2}}$.

In order to make a straightforward numerical integration of the equations possible, we close the equations by assuming that the vortex has a Gaussian shape and a given time-independent mass, thus imposing an algebraic relation between the spin and the moment of inertia.

Hence we assume that 


$$
h(x, y, t)=a_{v}(t) \exp \left(-\left(x^{2}+y^{2}\right) / r_{v}(t)^{2}\right),
$$

where $x$ and $y$ are local, Cartesian coordinates in the neighborhood of the origin of the coordinate system that is attached to the center of mass of the vortex [see Appendix E and relation (41)].

It follows that

$$
m=\int_{-\infty}^{\infty} \int_{-\infty}^{\infty} h(x, y, t) d x d y=\pi a_{v}(t) r_{v}(t)^{2}
$$

and

$$
m_{r^{2}}(t)=\int_{-\infty}^{\infty} \int_{-\infty}^{\infty} h(x, y, t)\left(x^{2}+y^{2}\right) d x d y=\pi a_{v}(t) r_{v}(t)^{4}
$$

Note that we consider the mass $m$ to be a time independent given parameter of the vortex.

Next we construct the contravariant geostrophic velocity and a cyclostrophic correction to this as follows.

To describe cyclostropic effects, we add inertia terms to the geostrophic balance $\breve{v}^{j} d \omega_{j i}=$ $-\gamma d h_{i}$,

$$
\breve{v}^{j} d \omega_{j i}=-\gamma d h_{i}-v^{j} \nabla_{j} v_{i} .
$$

With respect to local polar coordinates $(r, \phi)$ we find

$$
\breve{v}^{\phi}=\frac{\gamma}{a^{2} f r} \frac{\partial h}{\partial r}-\frac{1}{f}\left(\breve{v}^{\phi}\right)^{2},
$$

here $\breve{v}^{\phi}$ denotes the contravariant component of $\breve{v}$ in the direction of $\phi$.

Now we assume that the velocity field is almost geostrophic, i.e., that $\breve{v}^{\phi} \ll f$. On this condition we may use expression (55) as an iterative scheme for $\breve{v}^{\phi}$. As the first step in the iterative sequence, we take the geostrophic balance, i.e., the centrifugal acceleration, the second term on the right hand side, is neglected. The second step in the iterative sequence then is

$$
\breve{v}^{\phi}=\frac{\gamma}{a^{2} f r} \frac{\partial h}{\partial r}-\frac{\gamma^{2}}{a^{4} f^{3} r^{2}}\left(\frac{\partial h}{\partial r}\right)^{2} .
$$

With $r^{2}=x^{2}+y^{2}$ relation (56) can be applied to (51) to construct the geostrophic velocity of the Gaussian vortex corrected for the centrifugal acceleration of the geostrophic velocity.

The spin $\left\{\tau_{0} \| \rho \breve{v}\right\}$ can be evaluated for both the geostrophic Gaussian vortex and its cyclostrophically corrected counterpart. The spin is then expressed as a function of the amplitude $a_{v}$ and the radius $r_{v}$ of the vortex, but using (52) and (53), we can express it in terms of the total mass and moment of inertia. After tedious algebra, one finds

$$
\begin{aligned}
\left\{\tau_{0} \| \rho \breve{v}\right\}= & -\left(a _ { g } H \operatorname { c s c } ( \theta ) m \left(4 a_{g} C_{g c} H \csc (\theta)^{2} m^{5}+9 a_{g} C_{g c} H \pi \csc (\theta)^{2} m^{3} m_{r^{2}}+18 \Omega^{2} \pi a^{2} m^{2} m_{r^{2}}^{2}\right.\right. \\
& \left.\left.+72 \Omega^{2} \pi^{2} a^{2} m_{r^{2}}^{3}\right)\right) /\left(72 \Omega^{3} \pi^{2} a^{4} m_{r^{2}}^{3}\right),
\end{aligned}
$$

we have included the coefficient $C_{g c}$ : if we set $C_{g c}=0$, it gives the geostrophic result while setting $C_{g c}=1$ includes the cyclostrophic correction.

If we adopt formula (57) to express the spin in terms of mass and moment of inertia, then by doing so we eliminate spin as a variable and hence close the system of angular momentum equations. 


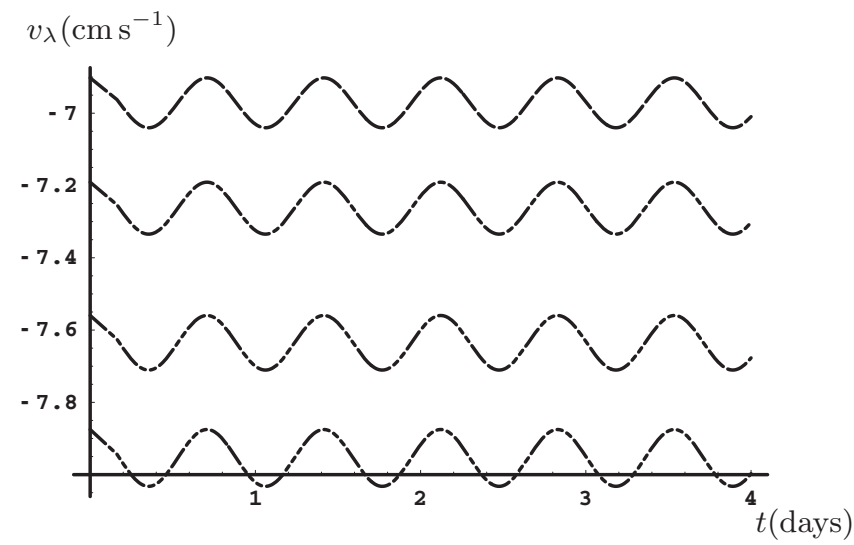

FIG. 2. Longitudinal velocities $v_{\lambda}\left(\mathrm{cm} \mathrm{s}^{-1}\right)$ as a function of time $t$ (days) of, from top to bottom, a cyclogeostrophic cyclone, a geostrophic cyclone, a geostrophic anticyclone, and a cyclogeostrophic anticyclone. All these vortices had a Gaussian profile and obey the full angular momentum equations (48)-(50). Spatially the vortices oscillate about a fixed latitude, in this case about $\theta=45^{\circ}$, while drifting westward.

\section{B. Numerical solutions}

As input for numerical integration of the equations we used representative values of the various parameters $\left(H=5 \mathrm{~km}, a_{v}(0)=0.1, r_{v}(0)=75 \mathrm{~km} / a, \Omega=7.29210^{-5} \mathrm{~s}^{-1}, a\right.$ $=6378 \mathrm{~km}, a_{g}^{\prime}=9.810^{-3} \mathrm{~m} / \mathrm{s}$ ).

Figure 2 shows longitudinal velocities $v_{\lambda}\left(\mathrm{cm} \mathrm{s}^{-1}\right)$ as a function of time $t$ (days), of, from top to bottom, a cyclogeostrophic cyclone, a geostrophic cyclone, a geostrophic anticyclone, and a cyclogeostrophic anticyclone. All these vortices were released at $\theta(0)=45^{\circ}$ with an initial westward velocity of $\lambda^{\prime}=-0.99 \varpi(0) /\left(2 \sin (\theta(0))\right.$. As we show in Sec. VIII B, $\lambda_{0}^{\prime}$ $=\varpi(0) /(2 \sin (\theta(0))$ is the angular velocity of stationary drift; see Eq. (63). In the solutions presented in Fig. 2, the velocity was slightly different from $\lambda_{0}^{\prime}$ and the velocity oscillates about its stationary value; for the motion as a whole this corresponds to notation about pure precession. In Sec. VIII E we explain why the various types of vortices have different velocities. Spatially the vortices oscillate about a fixed latitude, in this case about $\theta=45^{\circ}$, while they are all, independently of vortex type, drifting westward.

Figure 3 gives an overview of trajectories, each for the timespan of a few days, that result for a geostrophic anticyclone for various values of the initial value of the longitudinal angular velocity $\lambda^{\prime}$. For vortices of the other types the trajectories look similar. For $\lambda^{\prime}(0)=\lambda_{0}^{\prime}[$ Eq. (63)] we

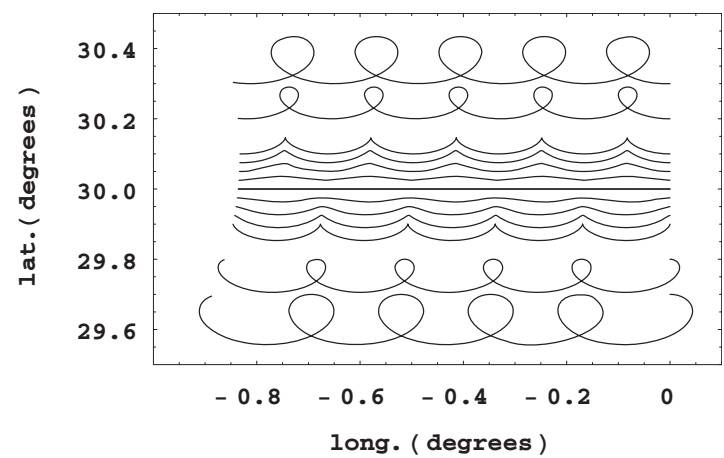

FIG. 3. Trajectories of several geostrophic anticyclones as found by numerical integration. At $t=0$ the vortices are released at several latitudes near $\theta=30^{\circ}$. They also have different initial longitudinal velocities. The vortex that starts at $30^{\circ}$ has initial velocity $\lambda_{0}^{\prime}$ as given by Eq. (63). Overall the vortices have initial velocities $(1+c) \lambda_{0}^{\prime}$, where, from bottom to top, $c=-3,-2,-1,-\frac{3}{4},-\frac{1}{2},-\frac{1}{4}, 0, \frac{1}{4}, \frac{1}{2}, \frac{3}{4}, 1,2,3$. 
observe steady westward drift. For initial values of $\lambda^{\prime}(0)$ close to $\lambda_{0}^{\prime}$ (deviations less then, say, $25 \%)$, trajectories show harmonic oscillation about a mean latitude. For larger deviations of $\lambda^{\prime}(0)$ from $\lambda_{0}^{\prime}$ the trajectories show nonharmonic oscillatory behavior. For deviations of $100 \%$ the trajectories show cusps at the north side on the northern hemisphere. On the southern hemisphere the cusps appear at the southern extrema of the trajectories. For deviations of $\lambda^{\prime}(0)$ from $\lambda_{0}^{\prime}$ exceeding $100 \%$ we find cycloidal trajectories. On the northern hemisphere the loops appear at the northern side of the trajectory; on the southern hemisphere they are at the southern side.

The amplitude of the latitudinal excursions of the trajectories depends on the initial latitude of the trajectories; it increases with decreasing latitudes, i.e., toward the equator $(\theta=0)$.

Both cusplike and cycloidal behavior were observed for solid disks moving along a solid sphere by McDonald: ${ }^{38}$ the motion of a disk consists of a periodic component superimposed on a drifting motion along a circle of constant latitude. However, the solid disk behaves essentially differently from vortices in that the direction of the drift, westward or eastward, for a disk is determined by the sense of spin: anticyclonic disks drift westward and cyclonic disks drift eastward. This is in sharp contrast to vortices, which drift westward regardless of spin orientation. Quantitatively the drift speed of a solid disk turned out to be an order of magnitude lower compared to the drift speed of, e.g., oceanic vortices.

\section{ANALYSIS}

\section{A. Decoupled form of the angular momentum equations}

Multiplying (49) by $\tan (\theta)$ and subtracting the result from (48), we readily find that

$$
\lambda^{\prime \prime}+\theta^{\prime}\left(\frac{\varpi}{\cos \theta}-2\left(1+\lambda^{\prime}\right) \tan \theta\right)=0 .
$$

A counterpart of this formula is found by adding $\cot \theta$ times Eq. (49) to Eq. (48), which leads to

$$
q \lambda^{\prime \prime}+q^{\prime}\left(1+\lambda^{\prime}\right)+\frac{\varpi^{\prime}}{\sin \theta}+q \theta^{\prime}\left(1+\lambda^{\prime}\right) \cot \theta=0 .
$$

As can be readily checked, $\cos (\theta)^{2}$ times (58) added to $\sin (\theta)^{2}$ times (59) returns Eq. (48), while $-2 \sin (2 \theta)$ times (58) added to $2 \sin (2 \theta)$ times (59) returns Eq. (49), so that we may conclude that the set of equations $\{(58),(59)\}$ is equivalent to the set $\{(48),(49)\}$ : one can be obtained from the other.

The set $\{(58),(59)\}$ is of a decoupled form, however, in the sense that the dynamical quantity $q$ no longer appears in (58). This decoupling is even stronger when some approximations, well applicable in the case of, e.g., oceanic vortices, are made as follows. From the scaling introduced in (46) it follows that the unit of $\lambda^{\prime}$ is radians per day so that for oceanic vortices $\lambda^{\prime} \ll 1$. Furthermore, it can be readily shown that for oceanic vortices the order of magnitude of the spin is $O(\varpi)=V L / a^{2} \Omega \approx 10^{-5} \cdots 10^{-4}$ so that, except in a small region about the equator $(\theta \approx 0)$, it holds $\varpi \ll \sin \theta$. Given these estimations, Eq. (58) may well be approximated by

$$
\lambda^{\prime \prime}-2 \theta^{\prime} \tan \theta=0 .
$$

From definition (46) of $q$ it readily follows that $O(q)=L^{2} / a^{2}$, where $L$ is radius of the vortex and $a$ is the radius of the planet. Hence $q \ll 1$. If we furthermore assume that the decay of vortices during a single day is much smaller than the strength of the vortex itself, i.e., $\varpi^{\prime} \ll \varpi$, then we may approximate Eq. (59) by

$$
q^{\prime}=0 .
$$

Under the assumption that $\lambda^{\prime} \ll 1$, the third angular momentum equation for vortices, (50), can be approximated by 


$$
\theta^{\prime \prime}-\cos (\theta) \varpi+\sin (2 \theta) \lambda^{\prime}=0
$$

In (60) the dynamical variables $q$ and $\varpi$ no longer occur, which shows that, within the framework of approximations that we have made, a direct relation exists between the spatial coordinates $\lambda$ and $\theta$; this means that orbits of a vortex are not sensitive to the strength $(\varpi)$ or geometrical features $(q)$ of the vortex.

Both sets of equations $\{(58),(59),(49)\}$ and $\{(60),(61),(62)\}$ are complicated and neither of them is a set of closed equations because in both sets we have more variables than equations. Yet a lot can be learned from these equations as follows.

Equation (61) shows that for slowly decaying vortices $\left(\boldsymbol{\varpi}^{\prime} \ll \varpi\right) q$ is approximately conserved and, if the spin $\varpi$ would be known, Eqs. (60) and (62) would rule the orbits of vortices. We see in Sec. VIII B that this immediately leads to a proper description on the sphere of the steady retrograde drift of vortices. Furthermore, we show that, except within a small region about the equator, this retrograde motion is stable, and we can construct oscillatory motion about the retrograde drift and determine its frequency.

We, furthermore, investigate the content of the approximation $\lambda^{\prime} \ll 1$ in Sec. VIII C, by taking a step back in the scheme of approximation and study drift $\left(\theta^{\prime}=0\right)$ without this approximation. Vortices with drift speeds that break the approximation $\lambda^{\prime} \ll 1$ do not seem to occur in nature, but an expression for $\lambda^{\prime}$ in case $\theta^{\prime}=0, \alpha_{\nu}=0, \alpha_{s}=0$ can be readily derived and it exemplifies the physical content of our angular momentum formalism.

\section{B. Intrinsic drift and the stability thereof}

A particular solution for Eqs. (60) and (62) is given by

$$
\theta^{\prime}=0, \quad \lambda^{\prime}=\lambda_{0}^{\prime}=\frac{\varpi}{2 \sin (\theta)} .
$$

This solution describes steady drift along a circle of constant latitude. Because, as was discussed in Sec. VI A, for regular, mainly geostrophic monopolar vortices, both cyclonic and anticyclonic ones, the sign of the spin $\varpi$ is always opposite to the sign of $\theta$, (63) shows that all regular monopolar vortices drift in retrograde sense independently of their orientation.

To investigate the stability of this intrinsic drift we expand $\theta, \lambda^{\prime}$, and $\varpi$ about their stationary values,

$$
\begin{gathered}
\theta(t)=\theta_{0}+\epsilon \theta_{1}(t)+O\left(\epsilon^{2}\right), \\
\lambda^{\prime}(t)=\frac{\varpi_{0}}{2 \sin \left(\theta_{0}\right)}+\epsilon \lambda_{1}^{\prime}(t)+O\left(\epsilon^{2}\right), \\
\varpi(t)=\varpi_{0}+\epsilon \varpi_{1}(t)+O\left(\epsilon^{2}\right) .
\end{gathered}
$$

Substitution of these expansions into Eqs. (60) and (62) readily leads to, in first order in $\epsilon$,

$$
\lambda_{1}^{\prime \prime}-2 \theta_{1}^{\prime} \tan \theta_{0}=0
$$

and

$$
\theta_{1}^{\prime \prime}+\varpi_{0} \cos \left(\theta_{0}\right) \cot \left(\theta_{0}\right)+\sin \left(2 \theta_{0}\right) \lambda_{1}^{\prime}=\cos \left(\theta_{0}\right) \varpi_{1} .
$$

Looking for solutions of the form

$$
\begin{aligned}
& \theta_{1}(t)=A \exp (\omega t), \\
& \lambda_{1}(t)=B \exp (\omega t),
\end{aligned}
$$




$$
\cos \left(\theta_{0}\right) \varpi_{1}(t)=C \exp (\omega t),
$$

one readily derives

$$
\omega^{2}=\frac{C}{A}-2 \cos \left(\theta_{0}\right)^{2}\left(2 \tan \left(\theta_{0}\right)^{2}+\lambda_{0}^{\prime}\right),
$$

where [see also (65)]

$$
\lambda_{0}^{\prime}=\frac{\varpi_{0}}{2 \sin \left(\theta_{0}\right)} .
$$

As long as $C / A$ is sufficiently small and $\left|\lambda_{0}^{\prime}\right| \ll 2 \tan \left(\theta_{0}\right)^{2}$,

$$
\omega \approx \pm 2 i \sin \left(\theta_{0}\right),
$$

holds, so that expressions (69) describe undamped oscillatory motions about the steady westward drift at a frequency equal to the inertial frequency, $2 \sin \left(\theta_{0}\right) \Omega$ in dimensional units. When

$$
2 \tan \left(\theta_{0}\right)^{2}+\lambda_{0}^{\prime} \approx 0,
$$

$\omega$ may easily become real and expressions (69) describe exponential growth of disturbances of steady solution (63). For realistic oceanic monopoles we have $\varpi_{0} \ll 1$. Hence in spite of the occurrence of $\sin \left(\theta_{0}\right)$ in the denominator of relation (71), relation (73) may be satisfied for small $\theta_{0}$. If $\theta_{0} \ll 1$, Eq. (73) reduces to

$$
\theta_{0}=\left(-\frac{1}{4} \varpi_{0}\right)^{1 / 3},
$$

which for a typical value $\varpi=-7 \times 10^{-5}$ gives $\theta_{0} \approx 1.5^{\circ}$, about $160 \mathrm{~km}$, on Earth. For such small values of $\theta$, Eqs. (63) predict a drift velocity of the same order of magnitude as the swirl velocity of oceanic vortices, which is another reason for suspecting instabilities of vortices in the equatorial region.

\section{Angular momentum equations and large drift velocity}

In Sec. VII we found that angular momentum dynamics predicts retrograde drift for slow $\left(\lambda^{\prime} \ll 1\right)$ regular monopolar vortices. We also found that the retrograde drift is stable as long as the vortices are not close to the equator.

In this section we take a step back in the approximation scheme and briefly investigate the meaning of the approximation $\lambda^{\prime} \ll 1$ to come to a somewhat better global understanding of the angular momentum equations.

The relation $\theta(t)=$ constant satisfies the first two of the three angular momentum equations, Eqs. (42) and (43), if, furthermore,

$$
\ddot{\lambda}=0, \quad \frac{d m_{r^{2}}}{d t}=0, \quad \text { and } \quad \frac{d}{d t}\left\{\tau_{0} \| \rho \breve{v}\right\}=0 .
$$

This solution corresponds to shape-preserving vortices that steadily translate along a fixed latitude. Their drift velocity is ruled by (44), which in the case at hand reduces to

$$
\dot{\lambda}(\dot{\lambda}+2 \Omega) m \sin (\theta)=(\Omega+\dot{\lambda})\left\{\tau_{0} \| \rho \breve{v}\right\},
$$

its dimensionless counterpart follows directly from Eq. (50), 
TABLE III. Correspondence of branches of Fig. 4 and the parameters $A$ and $B$ of Eq. (79).

\begin{tabular}{lcccc}
\hline \hline & sr & fr & si & fi \\
\hline A & 1 & 1 & -1 & -1 \\
B & 1 & -1 & 1 & -1 \\
\hline \hline
\end{tabular}

$$
\lambda^{\prime}\left(\lambda^{\prime}+2\right) \sin (\theta)=\left(\lambda^{\prime}+1\right) \varpi .
$$

This equation represents a balance between the torque exerted by the general gravity potential and the rate of change of spin due to the rotation of the coordinate system with respect to inertial space.

Relation (76) forms a quadratic equation for the drift speed $\dot{\lambda}$. Its quadratic character stems from the quadratic nature of the general potential, which in turn stems from the quadratic nature of the centrifugal potential in the angular frequency of the coordinate system. As we discuss in Appendix F 2 a, this quadratic nature of the centrifugal potential is reflected in the fact that there are two types of coordinates in which the general potential force vanishes identically.

\section{An overview of all modes}

The roots of (77) are

$$
\lambda_{ \pm}^{\prime}=\frac{\varpi-2 \sin (\theta) \pm \sqrt{\varpi^{2}+4 \sin (\theta)^{2}}}{2 \sin (\theta)} .
$$

As they are formulated in expression (78), the roots do not correspond to fixed types of monopoles. This can be seen with the help of Table II: if one would want to plot the intrinsic drift speed $\lambda^{\prime}$ of regular anticyclones as a function of $\theta$, for example, one would have to change the sign of $\varpi$ at $\theta=0$.

The following formula, a reformulation of expression (78), computes the drift velocity of a particular type of monopole:

$$
\lambda^{\prime}=\frac{-A|\varpi|-2|\sin (\theta)|+B \sqrt{\varpi^{2}+4 \sin (\theta)^{2}}}{2|\sin (\theta)|} .
$$

The type is characterized, as is summarized in Table III, by the parameters $A$ and $B: A=1$ for regular monopoles, i.e., for cyclones and regular anticyclones, and $A=-1$ for irregular anticyclones, while $B=1$ for slow monopoles and $B=-1$ for fast monopoles; the latter term denotes vortices that move retrogradely (westward) with about twice the angular frequency $\Omega$ with respect to the planet. These vortices would thus have angular frequency $\approx-\Omega$ with respect to inertial space. Therefore, they would experience more or less the same centrifugal force as vortices which are almost at rest with respect to the planet, for the latter have angular frequency $\Omega$, with respect to inertial space. This explains the existence of this second root. Such fast moving monopoles have never been observed in nature, however.

Roots (79) are plotted in Fig. 4 for all four types of monopoles. Any type is represented by a branch of the plot. Figure 4 represents roots (79) for $|\varpi|=0.1$, which is unrealistically large $\left(O(\varpi)=V L /\left(a^{2} \Omega\right) \approx 10^{-5} \cdots 10^{-4}\right)$. For realistic values of $|\varpi|$ the structure of the plot would be the same, but the curves would lie visually uncomfortably close to the lines $\theta=0, \lambda^{\prime}=-1$, and $\lambda^{\prime}=-2$.

Monopolar vortices observed in the terrestrial oceans all belong to the branch "sr" of the slow, regular monopoles, i.e., cyclones and regular (high-pressure) anticyclones. At this branch $\lambda^{\prime}$ is always negative for both cyclones and anticyclones at each of the hemispheres. This means that the intrinsic drift of all occurring monopolar vortices in the terrestrial oceans is westward. 


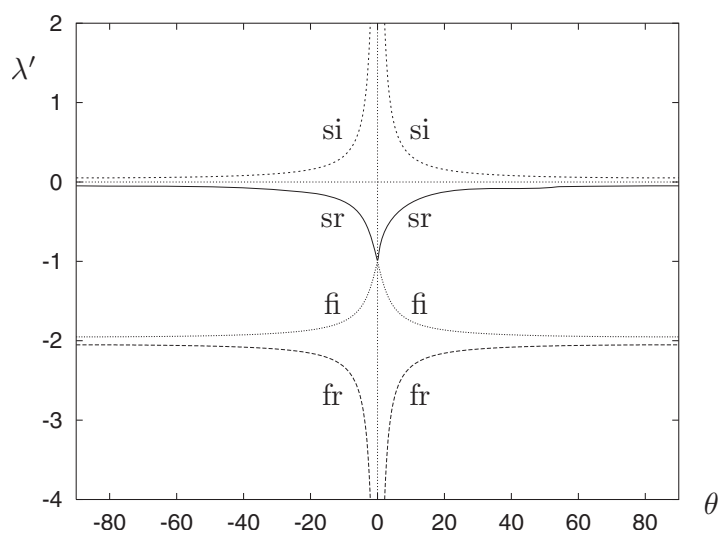

FIG. 4. The dimensionless drift speed $\lambda^{\prime}$ [solution of Eq. (77)] of monopoles as a function of geographical latitude $\theta$ for various modes: slow regular monopoles (sr), slow irregular anticyclones (si), fast regular monopoles (fr), and fast irregular anticyclones (fi)

Graphically the appearance of the curves in Fig. 4 is dominated by the equator, $\theta=0$. Slow, irregular anticyclones and regular fast monopoles show singular behavior at the equator. For all types of vortices, Eq. (79) predicts very large velocities near the equator.

\section{Limit of low drift speed}

We now inspect the limit of small drift speeds, which is valid for observed monopoles.

The dimensionless equation for drift speed (77) may be written as

$$
\lambda^{\prime}=\frac{1+\lambda^{\prime}}{1+\frac{1}{2} \lambda^{\prime}} \frac{\varpi}{2 \sin (\theta)} .
$$

In the limit of small drift speeds $\lambda^{\prime} \ll 1$, we recover Eq. (63).

Noteworthy is the fact that in the limit of low drift speed, the drift speed $\dot{\lambda}$ does not, at least not explicitly, depend on the rotation frequency $\Omega$ of the planet.

This may be understood as follows. At the limit $\dot{\lambda} \ll \Omega$ drift-induced general potential force (F8) is proportional to $\Omega$ because it is dominated by the cross term $\langle\nu, \omega\rangle$. The absolute velocity of the coordinate system $s$ is also dominated by the velocity of the planet $\omega$. Therefore, the rate of change of the spin vector of the vortex is approximately proportional to $\Omega$.

As a result in the limit of small drift speeds both sides of the drift balance are proportional to $\Omega$ and thus $\Omega$ does effectively not play a role in this balance, in this limit.

\section{The geostrophic balance: Covariant and conventional form}

In the geostrophic model of a monopole, it is assumed that the velocity field $\breve{v}$ is determined by the depth field $h$ of the reduced gravity single-layer model through the geostrophic balance. This balance states that Coriolis forces are balanced by pressure forces.

The physical Cartesian components, in $\mathrm{m} / \mathrm{s}$, of the geostrophic velocity are

$$
\breve{v}^{1^{*}}=-\frac{\gamma}{f} \frac{\partial h}{\partial y^{*}}, \quad \breve{v}^{2^{*}}=\frac{\gamma}{f} \frac{\partial h}{\partial x^{*}},
$$

where $x^{*}=a x, y^{*}=a y$, and $f=2 \Omega \sin (\theta)$. Hence the contravariant components $\breve{v}^{i}=\breve{v}^{i^{*}} / a$ are given by 


$$
\breve{v}^{1}=-\frac{\gamma}{a^{2} f} \frac{\partial h}{\partial y}, \breve{v}^{2}=\frac{\gamma}{a^{2} f} \frac{\partial h}{\partial x} .
$$

With this expression and with definition (F5) of $\tau_{0}$ with respect to local geodesic coordinates, we find for the monopolar vortex strength,

$$
\begin{aligned}
\left\{\tau_{0} \|(1+h) \breve{v}\right\} & =\frac{\gamma}{a^{2} \cdot f} \int(1+h)\left(x \frac{\partial h}{\partial x}+y \frac{\partial h}{\partial y}\right) d x d y=\frac{\gamma}{a^{2} \cdot f} \int\left(x \frac{\partial}{\partial x}+y \frac{\partial}{\partial y}\right)\left(h+\frac{1}{2} h^{2}\right) d x d y \\
& =-\frac{2 \gamma}{a^{2} f} \int h+\frac{1}{2} h^{2} d x d y+\frac{\gamma}{a^{2} f} \int\left(\left[x\left(h+h^{2}\right)\right]_{x=-\infty}^{\infty}\right) d y+\frac{\gamma}{a^{2} f} \int\left(\left[y\left(h+h^{2}\right)\right]_{y=-\infty}^{\infty}\right) d x \\
& =-\frac{2 \gamma}{a^{2} f} \int h+\frac{1}{2} h^{2} d x d y .
\end{aligned}
$$

The last step is valid if the vortex is sufficiently localized.

For the physical component $[\dot{\lambda}]$ of the drift speed in $\left(\mathrm{ms}^{-1}\right)$,

$$
[\dot{\lambda}]=a \cos (\theta) \dot{\lambda},
$$

we find therefore from (63) for a frictionless, slowly drifting, geostrophic monopole,

$$
[\dot{\lambda}]=-\frac{\gamma \cos (\theta)}{2 \Omega a \sin (\theta)^{2}} \frac{\int h+\frac{1}{2} h^{2} d x d y}{\int h d x d y}
$$

or

$$
[\dot{\lambda}]=-\beta_{0} R_{d}^{2}\left(1+\frac{\int h^{2} d x d y}{2 \int h d x d y}\right),
$$

where

$$
\beta_{0}=2 \frac{\Omega}{a} \cos (\theta) \quad \text { and } \quad R_{d}=\frac{\sqrt{\varepsilon_{\rho} a_{g} H}}{f}
$$

are the standard $\beta_{0}$ parameter and the equivalent-barotropic Rossby radius of deformation, respectively.

From formula (86) it follows that geostrophic anticyclones, having positive $h$, travel faster than long Rossby waves, while cyclones $(h<0)$ travel somewhat more slowly.

Under the transcription of notations-while we called the total depth of the shallow-water system $H \cdot(1+h),{ }^{25}$ called it just $H+\eta-$

$$
h \rightarrow \frac{\eta}{H},
$$

where $H$ is the depth of reference of the shallow-water system and $\eta$ the dimensional deviation from this depth, relation (85) is equivalent to relation (16) of Ref. 25.

\section{E. Effect of centrifugal correction on drift velocity}

In Sec. VIII D we saw that there is a difference in drift speed between cyclonic and anticyclonic geostrophic monopoles. In this subsection we show that this difference is amplified by centrifugal effects.

In terms of dimensional quantities, $h^{*}=H h, r=a r$, and $\breve{v}^{*}=a r \breve{v}^{\phi},(56)$ is 


$$
\breve{v}^{*}=\frac{a_{g}^{\prime}}{f} \frac{\partial h^{*}}{\partial r^{*}}-\frac{\left(a_{g}^{\prime}\right)^{2}}{f^{3} r^{*}}\left(\frac{\partial h^{*}}{\partial r^{*}}\right)^{2} .
$$

For Gulf Stream rings, $O\left(h^{*}\right)=500 \mathrm{~m}$ and $O\left(r^{*}\right)=100 \mathrm{~km}$, we have for $O\left(\breve{v}^{*}\right)$ a geostrophic value of $80 \mathrm{~cm} / \mathrm{s}$, corrected by a centrifugal correction of $9 \mathrm{~cm} / \mathrm{s}$, i.e., $11 \%$.

If we combine (56) with general expression (63) for slow drift, analogously to the derivation of (86), corrected geostrophic velocity (56) gives rise to a corrected expression for drift velocity (86),

$$
[\dot{\lambda}]=-\beta_{0} R_{d}^{2}\left(1+\frac{\int h^{2} r^{*} d r^{*}}{2 \int h r^{*} d r^{*}}\right)-\frac{1}{2} \beta_{0} R_{d}^{4} \frac{\int(1+h)\left(\frac{\partial h}{\partial r^{*}}\right)^{2} r^{*} d r^{*}}{\int h r^{*} d r^{*}} .
$$

The first term on the right hand side is equivalent to the geostrophic expression of the drift speed (86), although in (89) it is expressed with respect to polar coordinates and derived on condition of circular symmetry.

The centrifugal correction, the second term, is positive for cyclones $(h<0)$ and negative for regular anticyclones $(h>0)$. This means that the centrifugal effect increases the westward drift speed of regular anticyclones and decreases the drift speed of cyclones.

This is because the centrifugal effect diminishes the mass anomaly of regular anticyclones and enlarges the mass anomaly of cyclones. Thus, it diminishes the effect of the total potential force on anticyclones so that higher drift speeds are required to generate a potential force large enough to balance the change of spin with respect to inertial space. For cyclones the opposite is the case.

The orders of magnitude of the geostrophic and the centrifugal contribution to the drift speed, i.e., of the first and the second term of expression (89), are in the proportion of

$$
\frac{1}{2} \frac{R_{d}^{2}}{L^{2}}
$$

where $L=O\left(r^{*}\right)$. For Gulf Stream rings, where $L$ is twice to three times $R_{d}{ }^{25}$ this would amount to a correction of $6 \%-13 \%$. This explains published results from numerical experiments, based on reduced gravity, single-layer, shallow-water dynamics ${ }^{25}$ that showed that (regular) anticyclones drift systematically faster than predicted by geostrophic formula (86): Gulf-Stream ring-like anticyclones turned out to drift 5\%-9\% faster than predicted by geostrophic expression (86) of the drift speed.

\section{INTERPRETATION IN TERMS OF AN ANGULAR MOMENTUM DIAGRAM}

In this section we summarize the insights we have gained into the drift of vortices in a shallow fluid shell on a sphere. We do so by summarizing our results in terms of angular momentum vector diagrams. The formal link between the algebraic formalism and diagrams is discussed by Van der Toorn. ${ }^{36}$ Here we present the diagrams more directly.

In Fig. 5 we have represented schematically the angular momentum diagram for an anticyclonic monopole showing the mechanism of retrograde drift. At position $r$ we have indicated the net effective gravitational force $\mathbf{F}_{\mathbf{g}}$ which acts on the fluid shell as a whole as a result of the positive mass anomaly $m$ of the vortex that is at position $r$. Furthermore, we have indicated the associated effective torque $\mathbf{N}_{\mathrm{g}}=\mathbf{r} \times \mathbf{F}_{\mathrm{g}}$ and the spin $\mathbf{S}$ of the vortex. The vector $\boldsymbol{\omega}$ denotes the angular velocity vector of the solid planet with respect to inertial space, $\boldsymbol{\nu}$ the angular velocity vector of the vortex center of mass with respect to the planet, and $\mathbf{s}=\boldsymbol{\omega}+\boldsymbol{\nu}$ the angular velocity vector of the vortex with respect to inertial space.

The total angular momentum vector $\mathbf{J}$ of the vortex is the sum of an orbital momentum $\mathbf{L}_{\omega}$ induced by $\boldsymbol{\omega}$, the spin $\mathbf{S}$ of the vortex, and an orbital angular momentum $\mathbf{L}_{\boldsymbol{\nu}}$ induced by $\boldsymbol{\nu}$. The orbital momenta $\mathbf{L}_{\omega}$ and $\mathbf{L}_{\nu}$ come from $\boldsymbol{\omega}$ and $\boldsymbol{\nu}$ through the inertia tensor. This has been illus- 


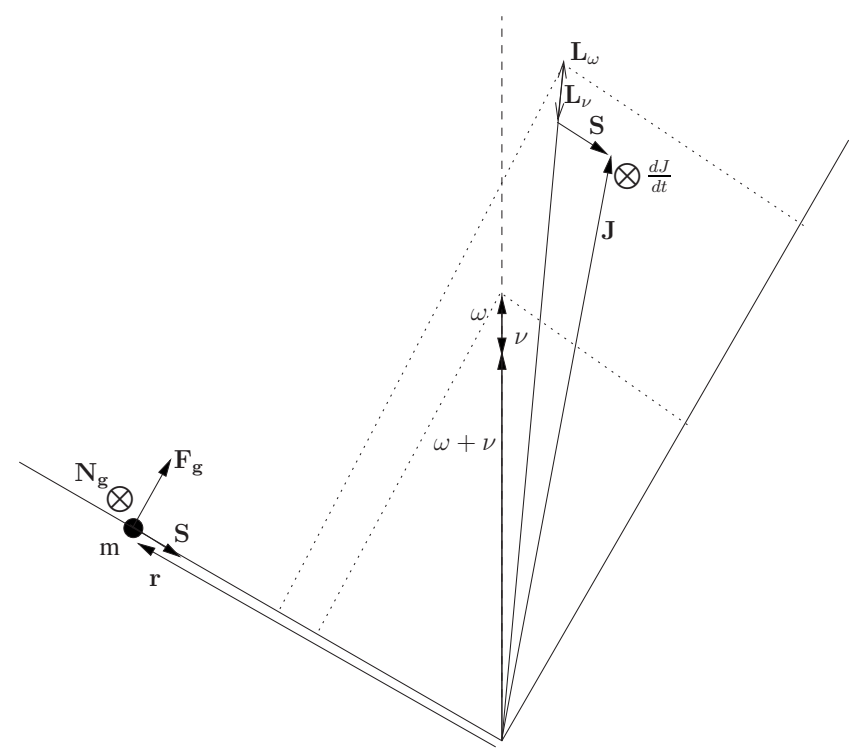

FIG. 5. Angular momentum diagram for an anticyclonic monopole showing the mechanism of retrograde drift. The encircled cross symbols represent vectors perpendicular to, and into, the plane of the paper.

trated in the figure for $\mathbf{L}_{\omega}$ as follows. The principal axes of the inertia tensor are the line along $\mathbf{r}$, through the vortex, and two lines perpendicular to it, one of which is indicated in the figure. To construct $\mathbf{L}_{\omega}, \boldsymbol{\omega}$ is decomposed with respect to the principal axes of the moment of inertia; this is indicated by dashed lines in Fig. 5. The components thus obtained are then scaled by the corresponding components of the moment of inertia to obtain the components of $\mathbf{L}_{\omega}$.

Because the moment of inertia about the axis through the vortex center is smaller than the moment about the other axes, the result for $\mathbf{L}_{\omega}$ is as sketched in the figure; note that for clarity, the scales used in the figure are rather arbitrary such that the qualitative structure of the diagram is clearly depicted.

Now, if we had considered instead of a vortex a hill of sand, stationary with respect to the solid planet, then $\mathbf{L}_{\omega}$ would have been all of the angular momentum of the hill. By hypothesis such a hill would be in equilibrium, i.e., the rate of change $\boldsymbol{\omega} \times \mathbf{L}_{\omega}$ of its orbital angular momentum $\mathbf{L}_{\omega}$ would exactly be compensated by the only available torque, namely, the torque $\mathbf{N}_{\mathbf{g}}$ exerted by the horizontal component of gravitation $\mathbf{F}_{\mathbf{g}}$, which in turn is induced by the slight deformation of the planet from a spherical shape. ${ }^{31,33}$ In fact, this deformation of the planet is a consequence of the equilibrium of the gravitation of the planet itself with its rotation.

In the case of a vortex, however, the spin $S$ adds to orbital angular momentum. Then, because $\boldsymbol{\omega} \times \mathbf{L}_{\omega}$ is in balance with $\mathbf{N}_{\mathbf{g}}$, the quantity $\boldsymbol{\omega} \times\left(\mathbf{L}_{\omega}+\mathbf{S}\right)$ is not in balance with $\mathbf{N}_{\mathbf{g}}$. For this reason a monopolar vortex cannot be stationary with respect to the planet. From the diagram it is easily conceived that $\boldsymbol{\omega} \times\left(\mathbf{L}_{\omega}+\mathbf{S}\right)$ is too large with respect to $\mathbf{N}_{\mathbf{g}}$.

For a shape-preserving vortex we have

$$
d \mathbf{J} / d t=(\boldsymbol{\omega}+\boldsymbol{\nu}) \times \mathbf{J} .
$$

Balance of $d \mathbf{J} / d t$ with the torque $\mathbf{N}_{\mathbf{g}}$ is established by adding an angular velocity vector $\boldsymbol{\nu}$ to $\boldsymbol{\omega}$; see Fig. 5. This makes $\mathbf{s}=\boldsymbol{\omega}+\boldsymbol{\nu}$ smaller than $\boldsymbol{\omega}$ and adds a contribution $\mathbf{L}_{\nu}$ to $\mathbf{J}=\mathbf{L}_{\omega}+\mathbf{S}+\mathbf{L}_{\nu}$ which also helps to reduce the rate of change of $\mathbf{J}$. With respect to the planet $\boldsymbol{\nu}$ is observed as the retrograde drift of the monopole.

It may serve understanding here to remark the following, with respect to the horizontal component of gravity, that is, experienced by an object at the surface of the planet, if it approximately corotates with the planet. If an object moves in an, e.g., moderate retrograde sense, the centrifugal force is decreased as compared to the case in which the object is stationary with respect 


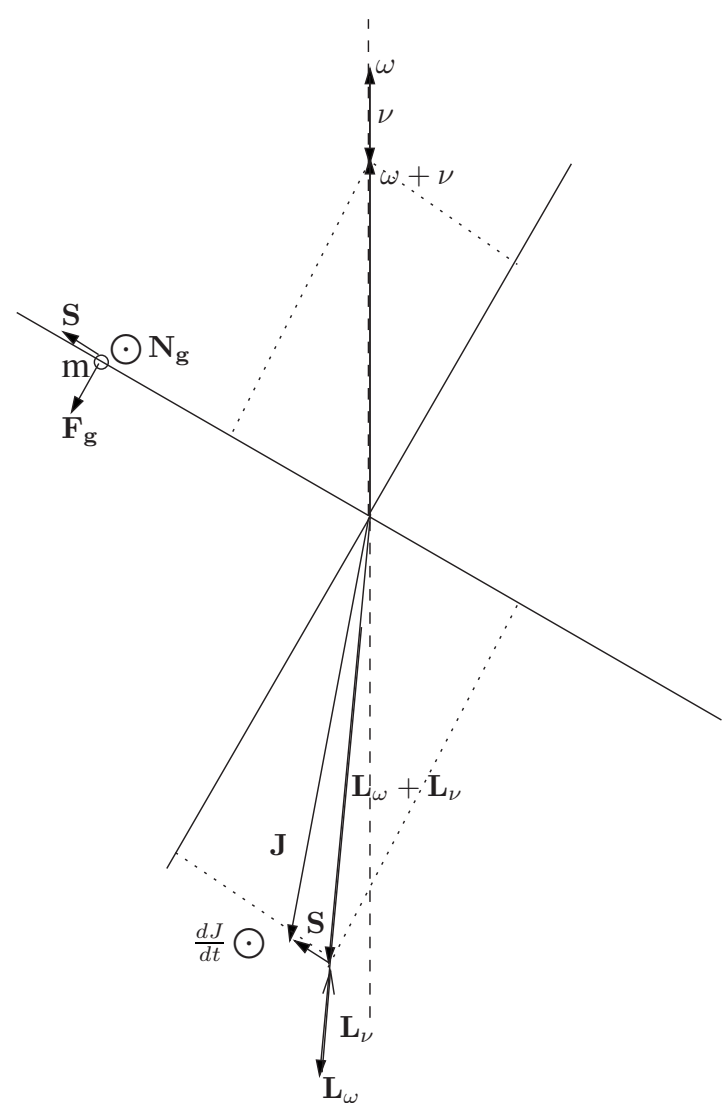

FIG. 6. Angular momentum diagram for a cyclonic monopole, showing that cyclones, too, drift in retrograde direction (westward). The encircled dot symbols represent vectors perpendicular to the plane of the paper and in the direction toward the reader.

to the surface of the planet. Hence the balance, that exists when the object is stationary with respect to the surface of the planet, between the horizontal component of the force of gravitation and the centrifugal force, is broken. As a result, an object with (positive) mass that moves in a moderate retrograde sense will experience a net poleward force along the surface of the planet, corresponding to a surplus of gravitational force, as compared to the partially compensating centrifugal force. Note that the centrifugal force here is defined with reference to a coordinate system that is fixed to the object, not to the planet. The model of the planetary gravity field applied in the present manuscript has been discussed in detail in Ref. 33.

An intriguing feature of the drift of monopolar vortices is that both regular anticyclones and cyclones drift in retrograde sense ("westward") in spite of the opposite orientation of their spins. Figure 6 reveals why this is so. The basic principle is that the total angular momentum of the fluid shell and the total torque of gravitation acting on the shell must balance. As we saw in Sec. V, the large contributions to these quantities from the passive background of this shell balance each other, and we do not depict these contributions in the diagram. Because for a cyclone $m<0$, the net force of gravitation induced by a cyclone is equatorward and the torque is eastward. Thus, not only the spin is of opposite orientation, compared to anticyclones but also the torque of gravitation is oppositely oriented as well and so is orbital angular momentum because both $m<0$ and $m_{r^{2}}$ $<0$. As the diagram shows, the effect of adding spin to orbital angular momentum would again be that $\boldsymbol{\omega} \times\left(\mathbf{L}_{\omega}+\mathbf{S}\right)$ would be too large to balance $\mathbf{N}_{\mathrm{g}}$ so that, to have $\mathbf{s} \times \mathbf{J}$ balance $\mathbf{N}_{\mathrm{g}}, \mathbf{s}=\boldsymbol{\omega}+\boldsymbol{\nu}$ should be smaller than $\boldsymbol{\omega}, \boldsymbol{\nu}$ is as indicated in the diagram, and hence again the drift of the vortex is retrograde (westward). 


\section{REMARKS ON THE DYNAMICS OF THE LOCAL VORTEX STRUCTURE AND THE RELATION OF MONOPOLES TO THE ROSSBY WAVE FIELD}

Time dependence of the moment of inertia $m_{r^{2}}$ was observed in the numerical study we presented in Sec. VII, which was based on closing the angular momentum equation by posing a Gaussian shape for the depth profile of the vortex. In reality the shape of a vortex is not Gaussian; in fact, the structure of the vortex is governed by dynamics of geophysical fluids, which in the case of monopolar vortices may involve coupling with, e.g., Rossby waves. The subject of coupling of monopolar vortices to waves on a $\beta$-plane has been subject of an extensive branch of the geophysical fluid dynamics literature. ${ }^{22,39-49}$ It has been found that with increasing intensity of the vortex, the dispersion decay of the vortex slows down substantially ${ }^{20,50-52}$ and perturbation analysis based solutions describing stationary, i.e., nonradiating, anticyclonic monopolar vortices on the $\beta$-plane have been published. ${ }^{26}$ The dynamics of the structure of the vortices on the rotating sphere lies outside the scope of the work presented here. A complete theory of geophysical monopoles on a sphere could consist of the theory on vortex drift on the rotating sphere presented in this paper on the one hand and coupled to a dynamics of the structure of the vortex on the other hand. Our formalism would then provide a coordinate system attached to the center of mass of the vortex, by calculating the drift speed of the vortex from its structure, while the dynamics of the structure could then be formulated with respect to this coordinate system. In this way the dynamics of drift and the dynamics of structure would be decoupled.

Such a decoupling is the more natural because in the present study we found, both in the numerical experiments and in our analytical study of angular momentum dynamics, that the drift trajectories of geophysical monopoles do not depend strongly on the details of the structure of the vortex.

\section{CONCLUSION}

Summarizing our findings, we state that, driven by their spin, both regular anticyclones and cyclones drift in retrograde sense (westward), quite in contrast with the behavior of rigid bodies, for which the orientation of precession does depend on the orientation of spin. Furthermore, calculation of drift speeds of oceanic monopoles from angular momentum fluid dynamics on basis of a Gaussian profile fitted to observed profiles produces the right order of magnitude of the observed drift speed of real vortices.

The result presented here is robust in the following sense. Any monopolar vortex, no matter its precise shape, obtains a precession with a strong westward (retrograde) component independent of its rotation sense provided it has a predominantly geostrophic dynamic balance, eventually corrected for cyclostrophic effects. This conclusion is inescapable from integral angular momentum balance. If a vortex does not appear to obey this law, it is because it moves with an ambient flow that masks the intrinsic drift, because it is arrested in the shear of the ambient flow such that the intrinsic drift of its mass center is precisely countered by the ambient flow, because it is not shape preserving, or because its intrinsic spin is not stationary. The latter two conditions apply to the transient state of a vortex; for instance, intensifying tropical cyclones or weakly nonlinear ocean eddies decaying because of Rossby wave radiation damping. In both cases the vortex may be deflected to a more northerly or southerly direction depending on the sign of its intrinsic spin. Arrest by the ambient flow may apply to the vortices of the giant planets which are strongly coupled to the shear pattern of the zonal jets. These vortices could easily adjust the position of their center of mass such that the intrinsic drift is offset by the zonal flow for which only a slight meridional displacement of the mass center from a latitude of vanishing zonal flow (maximum shear) is necessary. Strongly nonlinear mesoscale eddies in the central midlatitude oceans are probably the structures that come nearest to the ideal of freely drifting vortices that evolve over such a long timescale that their shape and intrinsic spin can be considered as approximately preserved, and in these regions they may move with an intrinsic speed that is larger than the time-averaged general circulation speed of their surroundings. 


\section{ACKNOWLEDGMENTS}

This work has been carried out as part of the Nonlinear Ocean Dynamics Program of the Department of Physical Oceanography of the Netherlands Institute for Sea Research.

\section{APPENDIX A: THE INNER PRODUCT OF THE MOMENTUM BALANCE WITH AN ARBITRARY VECTOR FIELD}

Because the operator $\delta / \delta t$ obeys the product rule, we have for an arbitrary vector field $\Psi$,

$$
\Psi^{p} \frac{\delta v_{p}}{\delta t}=\frac{\delta}{\delta t}\left(\Psi^{p} v_{p}\right)-v_{p} \frac{\delta}{\delta t} \Psi^{p}=\frac{d}{d t}\left(\Psi^{p} v_{p}\right)-v_{p} \frac{\delta}{\delta t} \Psi^{p},
$$

where we have emphasized the fact that $\delta / \delta t$ reduces to the simple form,

$$
\frac{d}{d t}=\frac{\partial}{\partial t}+v^{k} \frac{\partial}{\partial x^{k}}
$$

if it operates on a scalar field.

We combine the second term of (A1) with the inner product of $\Psi^{p}$ and the second and third term of (8), first splitting $d s$, then forming $\delta s / \delta t$,

$$
\begin{aligned}
\Psi^{p}( & \left.\frac{\partial s_{p}}{\partial t}+d s_{j p} v^{j}\right)-v_{p} \frac{\delta}{\delta t} \Psi^{p} \\
= & \Psi^{p}\left(\frac{\partial s_{p}}{\partial t}+\left(\nabla_{j} s_{p}-\nabla_{p} s_{j}\right) v^{j}\right)-v_{p} \frac{\delta}{\delta t} \Psi^{p} \\
& =\Psi^{p} \frac{\delta}{\delta t} s_{p}-\Psi^{p} v^{j} \nabla_{p} s_{j}-v_{p} \frac{\delta}{\delta t} \Psi^{p} \\
& =\frac{\delta}{\delta t}\left(\Psi^{p} s_{p}\right)-\left(v_{p}+s_{p}\right) \frac{\delta}{\delta t} \Psi^{p}-\Psi^{p} v^{j} \nabla_{p} s_{j} \\
& =\frac{d}{d t}\left(\Psi^{p} s_{p}\right)-\left(v_{p}+s_{p}\right) \frac{\partial}{\partial t} \Psi^{p}-\left(v_{p}+s_{p}\right) v^{q} \nabla_{q} \Psi^{p}-\Psi^{p} v^{j} \nabla_{p} s_{j} \\
& =\frac{d}{d t}\left(\Psi^{p} s_{p}\right)-\left(v_{p}+s_{p}\right) \frac{\partial}{\partial t} \Psi^{p}-v^{q}\left(s_{p} \nabla_{q} \Psi^{p}+\Psi^{p} \nabla_{p} s_{q}\right)-v_{p} v^{q} \nabla_{q} \Psi^{p} \\
& =\frac{d}{d t}\left(\Psi^{p} s_{p}\right)-\left(v_{p}+s_{p}\right) \frac{\partial}{\partial t} \Psi^{p}-v^{q} \mathcal{L}_{\Psi^{\prime}}\left(s_{q}-v^{p} v^{q} \nabla_{q} \Psi_{p} .\right.
\end{aligned}
$$

Note how the Coriolis tensor $d s_{j p}$ has been decomposed and distributed among the other tensors $d\left(\Psi^{p} s_{p}\right) / d t$ and $v^{q} \mathcal{L}_{\Psi}(s)_{q}$.

Making use of (A1) and (A2), for the inner product of (8), and an arbitrary vector field $\Psi$, we find Eq. (11).

\section{APPENDIX B: STRESS ONLY EXERTS A NET INTEGRAL TORQUE THROUGH THE SURFACE}

We consider the volume integral of the torque exerted by stress $(\Phi \in \operatorname{so}(3))$,

$$
\int_{V} \Phi^{k} \nabla_{j} \tau_{k}^{j} d V=\int_{V} \nabla_{j}\left(\Phi^{k} \tau_{k}^{j}\right)-\left(\nabla_{j} \Phi^{k}\right) \tau_{k}^{j} d V=\int_{V} \nabla_{j}\left(\Phi^{k} \tau_{k}^{j}\right) d V=\int_{S} \Phi^{k} \tau_{k}^{j} d S_{j}
$$

The second step holds because $\tau^{j k}$ is symmetric while, for $\Phi \in \operatorname{so}(3), \nabla_{j} \Phi_{k}$ is antisymmetric (18).

In the third step we apply Stokes' theorem. Equation (B1) shows that stress can only exert a net 
integral torque on the fluid shell through the boundary of the shell; internal stress within the volume of the shell does not contribute to the total net torque.

\section{APPENDIX C: PRESSURE DOES NOT CONTRIBUTE TO INTEGRAL ANGULAR MOMENTUM BALANCE}

To analyze the contribution of pressure to the total torque exerted on the outer fluid shell we set

$$
\nabla_{j} \tau_{k}^{j}=-\nabla_{k} p
$$

where $p$ denotes the pressure within the fluid. As we saw, only the tangential component $\nabla_{\bar{n}} p, \bar{n}$ $\in\{1,2\}$, of the pressure gradient (C1) is relevant for Eq. (20). In hydrostatic approximation, if we assume that the inner (lower) fluid layer does not carry a horizontal pressure gradient, the tangential component $\nabla_{\bar{n}} p$ is given by

$$
\nabla_{\bar{n}} p=a_{g}\left(\rho_{I I}-\rho_{I}\right) H \nabla_{\bar{n}} h, \quad \bar{n} \in\{1,2\} .
$$

Hence for $\bar{n} \in\{1,2\}$,

$$
\begin{aligned}
\int_{V} \Phi^{\bar{n}} \nabla_{\bar{n}} p d V & =\int_{S} \Phi^{\bar{n}} a_{g} H^{2}(1+h)\left(\rho_{I I}-\rho_{I}\right) \nabla_{\bar{n}} h d S=a_{g} H^{2}\left(\rho_{I I}-\rho_{I}\right)\left(\int_{S} \Phi^{\bar{n}} \nabla_{\bar{n}} h d S+\frac{1}{2} \int_{S} \Phi^{\bar{n}} \nabla_{\bar{n}} h^{2} d S\right) \\
& =0 .
\end{aligned}
$$

The conclusion that this integral vanishes stems from the fact that for any smooth function, $f$, $\Phi^{\bar{n}} \nabla_{\bar{n}} f$ is just the variation of $f$ along $\Phi$ and the integral of such a quantity over the whole surface of a sphere vanishes for continuous $f$ and $\Phi \in \operatorname{so}(3)$. Result (C3) represents an important merit of the integral angular momentum equation: the pressure does not contribute to it.

\section{APPENDIX D: TWO-DIMENSIONAL REPRESENTATION OF THREE-DIMENSIONAL ROTATIONS}

\section{Two-dimensional representation of generating vector fields and metric}

Because integral angular momentum (25) essentially is a two-dimensional equation in terms of the two dimensions of a spherical surface, we introduce a two-dimensional representation of vector fields that generate rotations along the spherical surface. These are just representations of the fields of Sec. III D with respect to spherical coordinates, restricted to the tangential dimensions of the spherical surface.

With respect to geographical coordinates, $x^{i}=(\lambda, \theta, r)$, defined by

$$
\begin{gathered}
c^{1}(\lambda, \theta, r)=r \cos (\lambda) \cos (\theta), \\
c^{2}(\lambda, \theta, r)=r \sin (\lambda) \cos (\theta), \\
c^{3}(\lambda, \theta, r)=r \sin (\theta),
\end{gathered}
$$

one readily finds for the contravariant components $\left(\mathbf{r}_{x}\right)^{j}$, related to the components $\left(\mathbf{r}_{x}\right)^{i^{\prime}}$ given in $\mathrm{Sec}$. III C by the rule of contravariant transformation,

$$
\left(\mathbf{r}_{x}\right)^{i^{\prime}}=\frac{\partial x^{i^{\prime}}}{\partial x^{j}}\left(\mathbf{r}_{x}\right)^{j}
$$

the following components:

$$
\mathbf{r}_{1}=(-\cos (\lambda) \tan (\theta), \sin (\lambda), 0)
$$




$$
\begin{aligned}
& \mathbf{r}_{2}=(-\sin (\lambda) \tan (\theta),-\cos (\lambda), 0), \\
& \mathbf{r}_{3}=(1,0,0) .
\end{aligned}
$$

By setting $r$ in (D1) equal to the radius $a$ of the sphere, we restrict to a two-dimensional spherical surface coordinatized by just the coordinates $x^{\mu}=(\lambda, \theta)$. Within such a spherical surface the fields $\mathbf{r}_{x}$ are simply described by

$$
\begin{gathered}
\mathbf{r}_{1}(\lambda, \theta)=(-\cos (\lambda) \tan (\theta), \sin (\lambda)), \\
\mathbf{r}_{2}(\lambda, \theta)=(-\sin (\lambda) \tan (\theta),-\cos (\lambda)), \\
\mathbf{r}_{3}(\lambda, \theta)=(1,0) .
\end{gathered}
$$

These vector fields again form a basis for the Lie algebra of rotations, so(3), endowed with products (16) and (17), where now $x^{1}=\lambda, x^{2}=\theta$.

For the metric of the spherical surface, represented with respect to the coordinates $(\lambda, \theta)$, we readily find

$$
g_{i j}=a^{2}\left(\begin{array}{cc}
\cos (\theta)^{2} & 0 \\
0 & 1
\end{array}\right)
$$

\section{Global inner product of vector fields}

An inner product,

$$
\langle\cdot \| \cdot\rangle: T M \times T M \rightarrow \mathbb{R},
$$

may be defined for vector fields $v$ and $w$ in the tangent space $T M$ of a spherical surface $M$ as follows:

$$
\langle v \| w\rangle=\frac{3}{8 \pi a^{4}} \int_{M} g_{i j} v^{i} w^{j} \sqrt{g} d x^{1} \wedge d x^{2},
$$

where $g$ is the determinant of the metric tensor $g_{i j}$ and $\sqrt{g} d x^{1} \wedge d x^{2}$ is the surface element of the surface. The scaling factor $3 /\left(8 \pi a^{4}\right)$ is chosen such that with respect to inner product (D12) the set $\left\{r_{\mu}\right\}$, (D7)-(D9), is a set of orthonormal vectors,

$$
\left\langle r_{\mu} \| r_{\nu}\right\rangle=\boldsymbol{\delta}_{\mu \nu}
$$

where $\boldsymbol{\delta}_{\mu \nu}$ is the Kronecker delta symbol. Relation (D13) may be viewed as the representation of metric (D12) with respect to our basis $\left\{r_{1}, r_{2}, r_{3}\right\}$ of so(3); hence the bold notation and Greek indices.

Let $f$ be a scalar field over the surface $M: f: M \rightarrow \mathbb{R}$. Then, from definition (D12) it immediately follows that $\left\langle f . r_{\mu} \| r_{\nu}\right\rangle=\left\langle r_{\mu} \| f . r_{\nu}\right\rangle$. We occasionally stress this symmetry of the inner product by use of the following "sandwich notation:"

$$
\left\langle r_{\mu} \| f r_{\nu}\right\rangle=\left\langle r_{\mu}|f| r_{\nu}\right\rangle
$$

\section{A totally skew-symmetric operator}

We consider the following nesting of the commutator and the inner product: 


$$
\langle[., .] \| .\rangle: T M \times T M \times T M \rightarrow \mathbb{R} .
$$

As indicated, arguments of this operator may be any vector fields over $M$. With respect to elements of so(3), however, (D15) is totally skew symmetric.

Indeed let $u, v, w \in \operatorname{so}(3)$. Then $u$ can be expressed as a linear combination of basis elements,

$$
u=\mathbf{u}^{\mu} r_{\mu} .
$$

The fields $v$ and $w$ may be represented analogously. Then, with multiplication rule (17) and metric (D13) of so(3),

$$
\langle[u, v] \| w\rangle=\mathbf{u}^{\mu} \mathbf{v}^{\nu} \mathbf{w}^{\lambda}\left\langle\left[r_{\mu}, r_{\nu}\right] \| r_{\lambda}\right\rangle=-\mathbf{u}^{\mu} \mathbf{v}^{\nu} \mathbf{w}^{\lambda} \boldsymbol{\epsilon}_{\mu \nu}{ }^{\kappa}\left\langle r_{\kappa} \| r_{\lambda}\right\rangle=-\mathbf{u}^{\mu} \mathbf{v}^{\nu} \mathbf{w}^{\lambda} \boldsymbol{\epsilon}_{\mu \nu}{ }^{\kappa} \boldsymbol{\delta}_{\kappa \lambda}=-\mathbf{u}^{\mu} \mathbf{v}^{\nu} \mathbf{w}^{\lambda} \boldsymbol{\epsilon}_{\mu \nu \lambda} .
$$

This demonstrates that (D15) is indeed totally skew symmetric with respect to elements of so(3). The nested brackets are related to the Levi-Civita symbol as

$$
\left\langle\left[r_{\lambda}, r_{\mu}\right] \| r_{\nu}\right\rangle=-\boldsymbol{\epsilon}_{\lambda \mu \nu}
$$

\section{APPENDIX E: A MOVING COORDINATE SYSTEM}

\section{Introduction}

In this section we set up a coordinate system for the spherical surface of the planet which may move with respect to the planet. The use of such a coordinate system is that one may attach its origin to an oceanic or atmospheric phenomenon, like a monopolar vortex, which may move with respect to the planet. As seen from the coordinate system, the phenomenon is then fixed at the origin $(x, y)=(0,0)$ of the system. The velocity of the coordinate system with respect to inertial space is represented by the vector field $s$ (29).

Oceanic vortices such as Gulf Stream Rings are rather small compared to the radius of the earth. In the region of such a vortex, therefore, the non-Euclidean metric of the spherical surface may be approximated by an Euclidean metric. It is a standard result of differential geometry that the best way to establish this approximation is by the use of a geodesic coordinate system. ${ }^{53} \mathrm{We}$ therefore construct our coordinate system to be of the geodesic type.

The spherical surface of the planet, which has radius $a$, is embedded in a three-dimensional Euclidean space $E_{3}$. Let $\left\{\bar{c}^{1}, \bar{c}^{2}, \bar{c}^{3}\right\}$ be Cartesian coordinates for $E_{3}$. Let the system $\bar{c}$ be attached to the planet; thus the system $\bar{c}$ is rotating with respect to inertial space with angular frequency $\Omega$. Let furthermore the $\bar{c}^{3}$-axis be the axis of this rotation. Let, finally, $\bar{c}^{i}=0$ denote the center of the planet. 
TABLE IV. The nonvanishing components of the metric and nonvanishing Christoffel symbols of spherical coordinate system (E3).

\begin{tabular}{lccc}
\hline \hline$g_{11}$ & $a^{2} \cdot \cos (y)^{2}$ & $\Gamma^{1}{ }_{12}$ & $-\tan (y)$ \\
$g_{22}$ & $a^{2}$ & $\Gamma^{2}{ }_{11}$ & $\frac{1}{2} \sin (2 y)$ \\
\hline \hline
\end{tabular}

By means of the matrices,

$$
R_{2}(x)=\left(\begin{array}{ccc}
\cos (x) & 0 & -\sin (x) \\
0 & 1 & 0 \\
\sin (x) & 0 & \cos (x)
\end{array}\right)
$$

and

$$
R_{3}(x)=\left(\begin{array}{ccc}
\cos (x) & -\sin (x) & 0 \\
\sin (x) & \cos (x) & 0 \\
0 & 0 & 1
\end{array}\right)
$$

we define coordinates $(x, y)$ for the spherical surface $M$ of radius $a$ by their relation to the Cartesian coordinates $\bar{c}^{i}$,

$$
\bar{c}=a \cdot R_{3}(\lambda) \cdot R_{2}(\theta) \cdot R_{3}(x) \cdot R_{2}(y) \cdot e_{1},
$$

where $e_{1}$ is the unit vector $(1,0,0)$.

The system $(x, y)$ looks like a geographical system, rotated such that its origin lies at geographical position $(\lambda, \theta)$. Its geometrical structure, i.e., its metric, etc., looks the same as that of the standard geographical system. The nonvanishing components of the metric and of the compatible affine connection are tabulated in Table IV. Because the system is of the geodesic type, all Christoffel symbols vanish at the origin of the system and the system behaves like a Cartesian system at the origin.

\section{The velocity of the coordinate system and that of the planet}

If coordinate system (E3) moves with respect to the planet, then the geographical coordinates of the origin of the system, $\lambda$ and $\theta$, depend on time. Such a time dependence implies a vector field $\bar{\nu}$, describing the velocity of the coordinate system with respect to the Cartesian system $\bar{c}$,

$$
\bar{\nu}=\frac{\partial \bar{c}}{\partial \lambda} \dot{\lambda}+\frac{\partial \bar{c}}{\partial \theta} \dot{\theta} .
$$

This vector field may be represented intrinsically, i.e., with respect to the system $(x, y)$ itself as well. The intrinsic, contravariant representation $\nu$ of the velocity field is related to $\bar{\nu}$ by

$$
\bar{\nu}^{i}=\frac{\partial \bar{c}^{i}}{\partial x^{j}} \nu^{j},
$$

i.e., by contravariant transformation.

It may be checked by direct computation that the following field obeys relation (E5),

$$
\nu(x, y)=\dot{\lambda} \hat{\omega}(x, y)-\dot{\theta} r_{2}(x, y),
$$

where $\hat{\omega}$ is defined as 


$$
\hat{\omega}(x, y)=\sin (\theta) r_{1}(x, y)+\cos (\theta) r_{3}(x, y) .
$$

The vector fields $\vec{e}_{n}$ are once again the base vectors of $\operatorname{so}(3)$, (D7)-(D9), $\mathbf{r}_{1}(x, y)=$ $(-\cos (x) \tan (y), \sin (x))$, etc. Note that in the current context these fields are fixed with respect to our moving coordinate system and that the field $\hat{\omega}$ may explicitly depend on time through $\theta$.

The velocity of the Cartesian system $\bar{c}$ with respect to an inertial frame of reference is described with respect to the system $\bar{c}$ itself by the vector field,

$$
\bar{\omega}=\Omega\left(-\bar{c}^{2}, \bar{c}^{1}, 0\right) .
$$

This field is tangent to the spherical surface $v^{q} \mathcal{L}_{\Psi}(s)_{q}$. It may be shown by direct transformation that, with respect to the system $(x, y)$, the restriction of $\bar{\omega}$ to $M$ is represented by

$$
\omega=\Omega \hat{\omega},
$$

which is the background for the choice of the symbol $\hat{\omega}$, (E7). The field $\omega$, (E9), is the intrinsic representation of the velocity of the planet with respect to inertial space.

The field $\nu$ may depend on time through the time dependence of $\dot{\lambda}, \dot{\theta}$, and/or $\hat{\omega}$. In turn the time dependence of $\hat{\omega}$ may be expressed analogously to (36) so that we have

$$
\frac{\partial}{\partial t} \nu=\ddot{\lambda} \hat{\omega}-\ddot{\theta} r_{2}+\dot{\lambda}[\nu, \hat{\omega}]
$$

\section{A physically meaningful basis for so(3)}

The standard base vector fields $r_{i}$ of so(3) do not have a further physical meaning. Therefore, we form another basis for so(3) which, through $\hat{\omega}$ and (E9), is connected to the physical circumstances.

We define

$$
\omega^{\dagger}=\cos (\theta) r_{1}-\sin (\theta) r_{3} .
$$

Then the tuple

$$
\left(\omega^{\dagger}, r_{2}, \hat{\omega}\right)
$$

forms a basis for so(3). This basis is orthonormal with respect to inner product (D11) and the basis elements obey the characteristic so(3) commutation relations,

$$
\left[\omega^{\dagger}, r_{2}\right]=-\hat{\omega}, \quad\left[r_{2}, \hat{\omega}\right]=-\omega^{\dagger}, \quad\left[\hat{\omega}, \omega^{\dagger}\right]=-r_{2} .
$$

We compute some commutators; from (E6), (E7), (E9), and (E13), it follows that

$$
[\nu, \omega]=\Omega \cdot \dot{\theta} \cdot \omega^{\dagger} .
$$

For the commutators of the velocity $s$, (29), with the new base vectors, we find

$$
\begin{aligned}
& {\left[\omega^{\dagger}, s\right]=(\Omega+\dot{\lambda}) r_{2}+\dot{\theta} \hat{\omega},} \\
& {\left[r_{2}, s\right]=-(\Omega+\dot{\lambda}) \omega^{\dagger},} \\
& {[\hat{\omega}, s]=-\dot{\theta} \omega^{\dagger} .}
\end{aligned}
$$




\section{APPENDIX F: LOCAL APPROXIMATE REPRESENTATIONS}

\section{Metric and Killing fields}

The metric of the coordinate system may be approximated locally in the neighborhood of the origin of our coordinate system by Taylor expansion of its components. Because our coordinate system is of the geodesic type, this local approximation of the metric of Table IV is a Cartesian metric,

$$
g_{i j}=a^{2} \delta_{i j}+O\left(x^{2}, y^{2}\right) .
$$

Likewise, we may approximate the base vector fields locally,

$$
\begin{aligned}
& \hat{\omega}=\sin (\theta) \tau_{0}+\cos (\theta) \tau_{1}+O\left(x^{3}, x^{2} y, y^{3}\right), \\
& r_{2}=-\tau_{2}+O\left(x^{2}, x y\right), \\
& \omega^{\dagger}=\cos (\theta) \tau_{0}-\sin (\theta) \tau_{1}+O\left(x^{3}, x^{2} y, y^{3}\right),
\end{aligned}
$$

where

$$
\tau_{0}=\left(\begin{array}{c}
-y \\
x
\end{array}\right), \quad \tau_{1}=\left(\begin{array}{l}
1 \\
0
\end{array}\right), \quad \tau_{2}=\left(\begin{array}{l}
0 \\
1
\end{array}\right) .
$$

The field $\tau_{0}$ is a generator of rotations in a flat plane around the origin $(x, y)=(0,0)$.

The fields $\tau_{1}$ and $\tau_{2}$ are generators of translations along such a plane. Fields (F5) span the algebra of isometries of a flat plane,

$$
\left[\tau_{1}, \tau_{2}\right]=0, \quad\left[\tau_{1}, \tau_{0}\right]=\tau_{2}, \quad\left[\tau_{0}, \tau_{2}\right]=\tau_{1} .
$$

From this we conclude that the multiplication table of so(3), (E13), cannot be reproduced from local approximations (F2)-(F4): as a result of the local approximation the algebra of Killing fields has been changed. Both the algebra of Killing fields and the metric are locally that of a flat plane.

\section{The general potential force}

\section{a. General form and properties}

If $s=\omega(\nu=0)$, the general potential $Y,(13)$, reduces to the geopotential $\bar{\Psi}$. One of the essential characteristics of the geopotential is that it is constant along the horizontal surface of reference. Hence for the gradient tangent to the surface of the planet, we have

$$
d\left(\left.Y\right|_{\nu=0}\right)=d\left(\phi-\frac{1}{2}\langle\omega, \omega\rangle\right)=0
$$

From (13), (F7), and (29), it readily follows that, in general,

$$
d \mathrm{Y}=-d\left\langle\nu, \omega+\frac{1}{2} \nu\right\rangle
$$

This expression clearly shows that there are two cases in which the general potential force $-d Y$ vanishes identically.

The first case is the case of a coordinate system attached to the planet, i.e., $\nu=0$; it is described by expression (F7). In this situation the coordinate system has a velocity $\omega$ with respect to inertial space and the horizontal centrifugal forces associated with the motion of the coordinate system exactly compensate the horizontal gravitational forces generated by the mass of the planet. Note that in this context "horizontal" means "tangent to the spherical surface of reference."

The second case is characterized by $\nu=-2 \omega$. In this case the coordinate system spins with respect to the planet in the direction opposite to the direction of the rotation of the planet with respect to inertial space. With respect to the planet the coordinate system has angular frequency $-2 \Omega$. With respect to inertial space therefore the angular frequency of the coordinate system is 
exactly equal to $-\Omega$. Therefore, as far as centrifugal forces are concerned, this situation is equivalent to the situation $\nu=0$, described above, and the total potential force $-d \Upsilon$ vanishes identically again.

\section{b. Local appearance}

By direct computation it can be shown that, with respect to the coordinate system of Appendix E 1, the force $(-d \Upsilon)^{i}$ generated by the general potential $Y$ is locally of the following form:

$$
(-d \Upsilon)^{i}=g^{i j} d\left\langle\nu, \omega+\frac{1}{2} \nu\right\rangle_{j}=\dot{\lambda}(\dot{\lambda}+2 \Omega) \sin (\theta) S^{i}+\dot{\theta}(\dot{\lambda}+\Omega)\left(\sin (\theta) \tau_{1}+\cos (\theta)\left(\begin{array}{l}
y \\
x
\end{array}\right)\right)-\dot{\theta}^{2} x \tau_{1},
$$

where

$$
S^{i}=\sin (\theta)\left(\begin{array}{l}
x \\
y
\end{array}\right)-\cos (\theta)\left(\begin{array}{l}
0 \\
1
\end{array}\right),
$$

and we have made use of the local field $\tau_{1}$, (F5). Force (F9) is associated with the motion $\nu$, (E6), of the coordinate system with respect to the rotating planet through $\dot{\lambda}$ and $\dot{\theta}$ and with the motion of the planet with respect to inertial space through $\Omega$.

Of course, as our formalism is completely two dimensional, expression (F9) only describes horizontal components of the forces, i.e., components that are tangent to the surface of the planet.

With longitudinal motions, $\dot{\theta}=0$ along curves of constant geographical latitude $\theta$, a force described by $S^{i},(\mathrm{~F} 10)$, is associated. In turn this field $S^{i}$ is composed of a part which points away from the origin and a part which points toward the geographical equator. These parts of $S^{i}$ correspond precisely to the parts of the local decomposition of the field $\hat{\omega}$, (F2). The first part, for example, is associated with the first part of $\hat{\omega}$, (F2), i.e., with rotations about the local origin of our moving coordinate system.

The latitudinal motion of the coordinate system $(\dot{\theta} \neq 0)$ only results in (longitudinal) potential forces if $\dot{\lambda}+\Omega \neq 0$. This may be understood from the fact that if $\dot{\lambda}+\Omega=0$, then a latitudinal motion $\dot{\theta}$ corresponds to a motion along a great circle on an inertial sphere, which is a natural motion (provided the particle is in some way constrained to the spherical surface).

\section{Local evaluation of the inner products over so(3)}

If the phenomenon under consideration is localized, i.e., if the fields $h$ and $\breve{v}$ associated with the phenomenon differ significantly from zero only in the neighborhood of the coordinate system, then inner products $\langle\cdot \| \cdot\rangle$ which have one of these fields in their arguments may be evaluated locally.

According to expansion (F1) the metric of the coordinate system of Appendix E 1 may, in the neighborhood of the origin, be approximated by a Cartesian one. Therefore, we may locally approximate the metrical structure of the spherical horizontal surface of reference by a Euclidean structure.

With the help of expansion (F1), expressions of the form $\langle a \| b\rangle$, (D12), can locally be evaluated as

$$
\langle a \| b\rangle=\frac{3}{8 \pi} \int \delta_{i j} a^{i} b^{j} d x d y .
$$

Because the total scaling factor $3 /(8 \pi)$ arises in all terms of basic angular momentum (28), it is convenient to define a local variant of the inner product which does not contain this scaling factor, 


$$
\{a \| b\}=\int \delta_{i j} a^{i} b^{j} d x d y .
$$

The symbol $\delta_{i j}$ is again the Kronecker delta symbol, just like in expression (F11).

We now introduce the Euclidean metric $\Delta_{i j}$. In terms of this tensor our local counterpart (F12) of the inner product $\langle\cdot \| \cdot\rangle$, (D12) may be written in covariant form as

$$
\{a \| b\}=\int \Delta_{i j} a^{i} b^{j} \sqrt{\Delta} d x^{1} \wedge d x^{2},
$$

where $\Delta$ is the determinant of $\Delta_{i j}$.

Occasionally we use a local counterpart of sandwich notation (D14); i.e., if $f$ is a scalar field, we have

$$
\{v \| f w\}=\{v|f| w\} .
$$

${ }^{1}$ D. B. Olson, Annu. Rev. Earth Planet Sci. 19, 283 (1991).

${ }^{2}$ P. L. Richardson, Prog. Oceanogr. 31, 1 (1993).

${ }^{3}$ P. Richardson, A. Bower, and W. Zenk, Prog. Oceanogr. 45, 209 (2000).

${ }^{4}$ K. Emanuel, Annu. Rev. Earth Planet Sci. 31, 75 (2003).

${ }^{5}$ J. C. L. Chan, Annu. Rev. Fluid Mech. 37, 99 (2005).

${ }^{6}$ P. S. Marcus, Annu. Rev. Astron. Astrophys. 31, 523 (1993).

${ }^{7}$ T. E. Dowling, Annu. Rev. Fluid Mech. 27, 293 (1995).

${ }^{8}$ A. L. Piro and L. Bildsten, Astrophys. J. 619, 1054 (2005).

${ }^{9}$ M. V. Nezlin, A. Y. Rylov, A. S. Trubnikov, and A. V. Khutoretskii, Geophys. Astrophys. Fluid Dyn. 52, 211 (1990).

${ }^{10}$ R. Morales-Juberías, A. Sánchez-Lavega, J. Lecacheux, and F. Colas, Icarus 157, 76 (2002).

${ }^{11}$ R. Morales-Juberías, A. Sánchez-Lavega, J. Lecacheux, and F. Colas, Icarus 160, 325 (2002).

${ }^{12}$ V. M. Kamenkovich, M. N. Koshlyakov, and A. S. Monin, Synoptic Eddies in the Ocean (Reidel, Dordrecht, 1986).

${ }^{13}$ A. L. Gordon, J. Geophys. Res. 91, 5037 (1986).

${ }^{14}$ D. B. Olson and R. J. Evans, Deep-Sea Res. 33, 27 (1986).

${ }^{15}$ D. A. Byrne, A. L. Gordon, and W. F. Haxby, J. Phys. Oceanogr. 25, 902 (1995).

${ }^{16}$ M. W. Schonten, W. P. M. De Ruijter, P. J. Van Leeuwen, and J. R. Lutjeharms, J. Geophys. Res. 105, 21,913 (2000).

${ }^{17}$ R. Morrow, F. Birol, D. Griffin, and J. Sudre, Geophys. Res. Lett. 31, L24311 (2004).

${ }^{18}$ D. B. Chelton, M. G. Schlax, R. M. Samelson, and R. A. de Szoeke, Geophys. Res. Lett. 34, L15606 (2007).

${ }^{19}$ D. L. Luce and T. Rossby, J. Geophys. Res. C 113, C05011 (2008).

${ }^{20}$ J. C. McWilliams and G. R. Flierl, J. Phys. Oceanogr. 9, 1155 (1979).

${ }^{21}$ D. Nof, J. Phys. Oceanogr. 11, 1662 (1981).

${ }^{22}$ P. D. Killworth, J. Phys. Oceanogr. 13, 368 (1983).

${ }^{23}$ D. Nof, J. Mar. Res. 41, 399 (1983).

${ }^{24}$ G. P. Williams and T. Yamagata, J. Atmos. Sci. 41, 453 (1984).

${ }^{25}$ B. Cushman-Roisin, E. P. Chassignet, and B. Tang, J. Phys. Oceanogr. 20, 758 (1990).

${ }^{26}$ J. Nycander and G. G. Sutyrin, Dyn. Atmos. Oceans 16, 473 (1992).

${ }^{27}$ P. J. van Leeuwen, J. Phys. Oceanogr. 37, 2316 (2007).

${ }^{28}$ R. Salmon, Annu. Rev. Fluid Mech. 20, 225 (1988).

${ }^{29}$ W. T. M. Verkley, J. Atmos. Sci. 47, 2453 (1990).

${ }^{30}$ D. Müller, Phys. Rev. A 45, 5545 (1992).

${ }^{31}$ J. Nycander, Plasma Phys. Rep. 22, 771 (1996).

${ }^{32}$ J. Pedlosky, Geophysical Fluid Dynamics (Spinger-Verlag, New York, 1987).

${ }^{33}$ R. van der Toorn and J. T. F. Zimmerman, Geophys. Astrophys. Fluid Dyn. 102, 349 (2008).

${ }^{34}$ B. A. Dubrovin, A. T. Fomenko, and S. P. Novikov, Modern Geometry, Methods and Applications, Graduate Texts in Mathematics (Springer-Verlag, New York, 1992), Pt. 1.

${ }^{35}$ E. Noether, Nachr. Ges. Wiss. Goettingen, Math.-Phys. K1. 2, 235 (1918).

${ }^{36} \mathrm{R}$. Van der Toorn, Ph.D. thesis, University of Utrecht, 1997.

${ }^{37}$ J. D. Logan, Applied Mathematics, 2nd ed. (Wiley, New York, 1997).

${ }^{38}$ N. R. McDonald, Geophys. Astrophys. Fluid Dyn. 87, 253 (1998).

${ }^{39}$ J. Adem, Tellus 8, 364 (1956).

${ }^{40}$ G. R. Flierl, J. Phys. Oceanogr. 7, 365 (1977).

${ }^{41}$ J. C. McWilliams, Rev. Geophys. 23, 165 (1985)

${ }^{42}$ G. R. Flierl, Annu. Rev. Fluid Mech. 19, 493 (1987).

${ }^{43}$ G. R. Flierl and K. Haines, Phys. Fluids 6, 3487 (1994).

${ }^{44}$ M. K. Davey and P. D. Killworth, J. Phys. Oceanogr. 14, 1047 (1984).

${ }^{45}$ G. I. Bell, Phys. Fluids A 2, 575 (1990).

${ }^{46}$ J. C. McWilliams, in Proceedings of the International School of Physics "Enrico Fermi," edited by A. R. Osborne 
(Elsevier, New York, 1991).

${ }^{47}$ G. K. Korotaev and A. B. Fedotov, J. Fluid Mech. 264, 277 (1994).

${ }^{48}$ G. K. Korotaev, Surv. Geophys. 18, 567 (1997).

${ }^{49}$ N. R. McDonald, J. Fluid Mech. 361, 237 (1998).

${ }^{50}$ G. G. Sutyrin and I. G. Yushina, Izvestiya Akademii Nauk SSSR 4, 52 (1986) [ Fluid Dyn. 21, 550 (1986)].

${ }^{51}$ G. G. Sutyrin, Sov. Phys. Dokl. 32, 791 (1987).

${ }^{52}$ G. G. Sutyrin, Izvestiya Akademii Nauk SSSR 2, 68 (1988)] [ Fluid Dyn. 23, 215 (1988).

${ }^{53}$ H. Stephani, General Relativity, 2nd ed. (Cambridge University Press, Cambridge, 1990). 